\title{
ENERGY UTILIZATION OF YAM BEAN (Pachyrhizus erosus L. Urban) GENOTYPES IN ILOCOS NORTE, PHILIPPINES
}

\author{
Jocelyn A. Bernabe \\ Mariano Marcos State University, College of Agriculture, Food \& Sustainable Development \\ Dingras, Ilocos Norte, Philippines
}

\begin{abstract}
A field experiment was conducted in three sites of Ilocos Norte: to evaluate the growth and yield performance of yam bean genotypes grown at different sites; determine the nutrient-use efficiency of yam bean genotypes grown at varying fertilizer treatments; determine the energy cost of producing different yam bean genotypes with fertilizer treatments in different growing sites; and, compute for the cost and return analysis of the different yam bean genotypes grown at varying fertilizer treatments and sites in Ilocos Norte.The experiment was laid out in split-plot design with fertilizer treatments (control, organic, $50 \%$ organic $+50 \%$ inorganic, inorganic) as the main-plot factors and genotypes (G1, G2, G3, G4 and G5) as the sub-plot. Results were tested and compared across three sites. Generally, fertilizer significantly affected yield and yield contributing characters in all sites but not all with genotypes. To improve yield and other plant characters as well as enhance soil fertility conditions, the use of organic fertilizer can be done since this is the cheapest, locally available, energy efficient and gives high returns.
\end{abstract}

Keywords: yambean, genotypes, energy utilization

\section{INTRODUCTION}

Yam bean is one of the Neotropical legume genera with edible tuberous roots. It is extensively cultivated, both as a garden crop, and on a large scale for export.

In the Philippines, this crop is popularly grown particularly in Luzon areas specifically in northeastern areas like Ilocos Norte, produces high root yields of 25-40 $\mathrm{t} \mathrm{ha}^{-1}$ to as high as $60 \mathrm{t} \mathrm{ha}^{-1}$, and seed or grain yield of 4-5 $\mathrm{t} \mathrm{ha}^{-1}$ (BAS, 2005). Currently, the area planted is not so wide, 24.30 ha (BAS POC Ilocos Norte, 2009) although it is periodically increasing due to attention being given because of its potential as source of additional income especially in its processed forms.

The Bureau of Agricultural Statistics Provincial Office (BASPO) of Ilocos Norte noted that there are different genotypes being grown with variable sizes and shapes at different sites in Ilocos Norte. Specifically, there are five genotypes observed being grown and sold in the local market, and in other provinces and regions that have not been identified and characterized for maximum yields and adaptability under varying growing conditions.

Yam bean root contains 32\% soluble sugars and $15 \%$ starch as storage carbohydrates on dry basis (Paul and Chen, 1988). The functional properties of yam bean starch, allows it to be used as potential source of starch (Melo et al., 2003

The seeds are characterized by high oil (20-28\%) and protein (23-34\%) contents. Seed oil contains high concentrations of palmitic (25-30\% of the total fatty acids), oleic (21-29\%), and linoleic acids (35-40\%) (Gruneberg et al., 1999).

The mature seeds contain up to $26 \%$ protein and $30 \%$ vegetable oil - a composition comparable to ground nut and cotton seed oils. However, the mature seeds contain up to $0.5 \%$ rotenone (an isoflavonoid), an insecticidal compound that makes them inedible but this secondary metabolite can

Corresponding Author: bernabejocelyn80@yahoo.com 
prevent harmful insects in vegetable fields. (Villar and Valio, 1994).

Organic farming is not only energy efficient, as it was also found to be equally or slightly more productive. Aside from improving the fertility status of the soil, organic fertilizer sources could be locally available and does not require much energy for the handling and processing. The FAO stressed that organic farming fights hunger, tackles climate change and is good to farmers, consumers and the environment because of its non-reliance to fossil fuel. It uses locally available resources with minimal agro-ecological stresses and is costeffective (Burcher, 2007).

Yam bean genotypes generally survive in all types of soil characteristics, but respond well to the addition of fertilizer materials. In addition, the crop shows favorable response to added nutrient inputs (Sorensen, 1990).

In Ilocos Norte, yam bean farmers usually apply inorganic fertilizer to their yam bean plants and it was observed based on record that the yield increased to $47.67 \mathrm{mt} \mathrm{ha}^{-1}$ (Table 1) as compared to yield during the last 5 years which was $15-20 \mathrm{mt}$ ha $^{-1}$ (DA PAO, 2009).
Energy consumption per unit area in agriculture is directly related with the development of technological level and production. The inputs such as fuel, electricity, machinery, seed, fertilizer and chemical take significant share of the energy supplies to the production system in modern agriculture. However, some problems in agricultural production have been faced due to mainly high level dependency on fossil energy. Improving the end-use energy efficiency is one of the most effective ways to reduce energy consumption in the industrial, commercial, transportation, utility, residential and agricultural sectors and their associated pollutant emissions (Dyer and Desjardins, 2003).

With the increasing demand due to the benefits from the crop, there is a need to clearly identify and evaluate these existing genotypes as to where they could fit in for optimum production.

This study was conducted to evaluate the growth and yield performance of yam bean genotypes grown at different sites; the energy cost of producing; and, compute for the cost and return analysis of the different yam bean genotypes grown at varying fertilizer treatments andsites in Ilocos Norte.

\section{CONCEPTUAL FRAMEWORK OF THE STUDY}

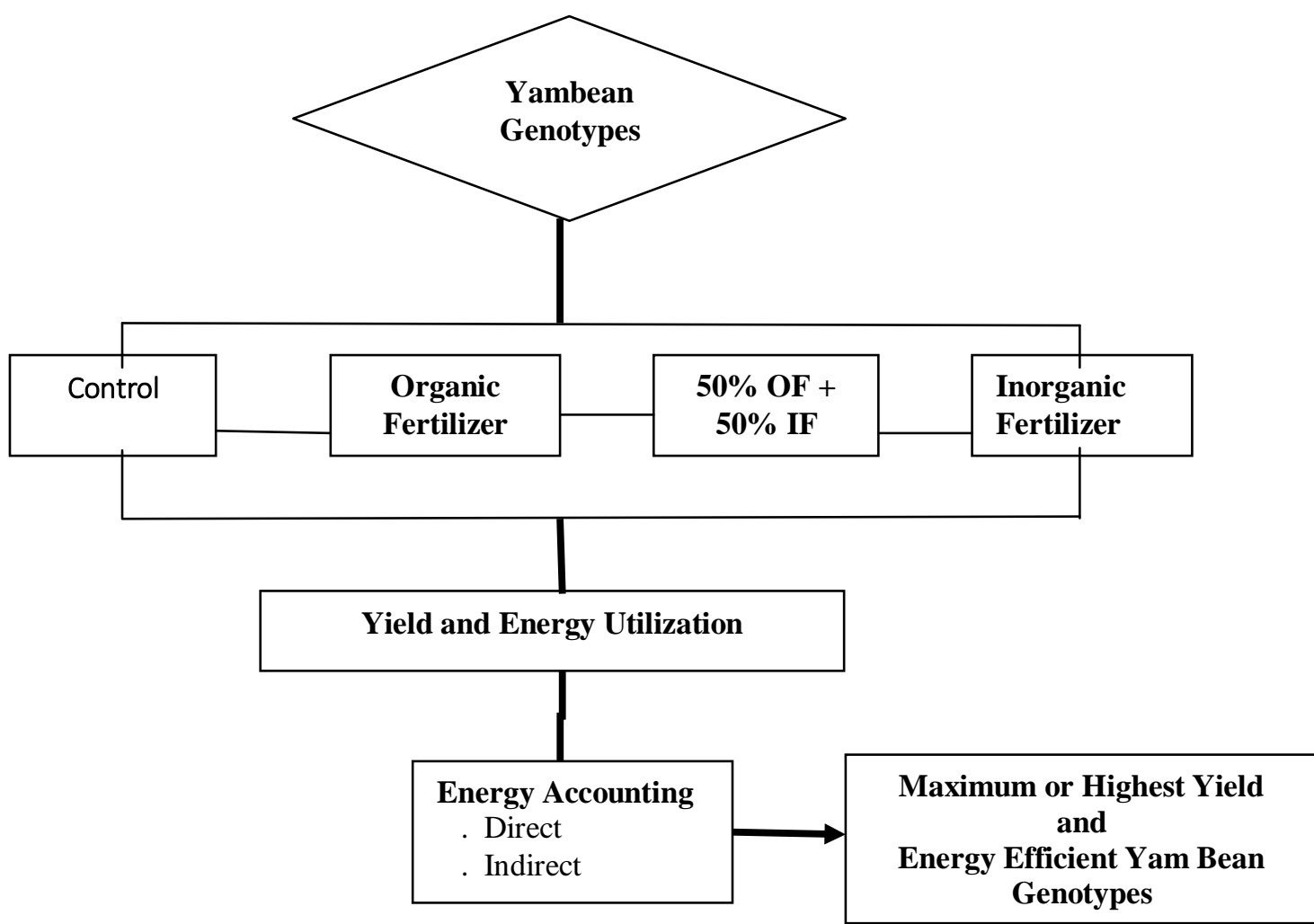




\section{MATERIALS AND METHODS}

Prior to the conduct of the study, a survey was done on the areas where yam bean is commonly grown (Table 1).

The experiment was conducted in three sites of Ilocos Norte, wherein each represents three soil series commonly grown to yam beans and are representative of the entire province.It was laid out in a Split-Plot Design in each site with fertilizer treatments $(F)$ in the mainplot and the yam bean genotypes $(\mathrm{G})$ in the subplot. The total area for each site was $750 \mathrm{~m}^{2}$.
Selected tubers of the different genotypes were planted to produce seeds, 6 months before the establishment of the experiment.

During the early vegetative stages of the crops, slight presence of thrips was observed, so Tamaron ${ }^{\circledR}$ insecticide was sprayed using the recommended dosage to prevent the multiplication and spread of the pests.

Harvesting was done by manually pulling or uprooting the roots by hand or digging tools.

Table 1. Volume of production, area and yield of yam bean in Ilocos Norte (January-June, 2008-2009)

\begin{tabular}{cc|c|c|c|c}
\hline \multicolumn{2}{c|}{ PRODUCTION $(\mathrm{mt})$} & \multicolumn{2}{c}{ AREA (ha) } & \multicolumn{2}{c}{ YIELD $\left(\mathrm{mt} \mathrm{h}^{-1}\right)$} \\
\hline 2008 & 2009 & 2008 & 2009 & 2008 & 2009 \\
185.27 & 186.40 & 24.20 & 24.30 & 47.66 & 47.67 \\
\hline
\end{tabular}

Source:BAS POC Ilocos Norte, 2009

The data used for this experiment and the computation of these parameters were recorded from the pre-establishment of the crop until harvesting. The author utilized the standard formula set by PCARRD 1978 for each. In terms of the energy, the procedure in the analysis and the energy coefficients of the materials and activities were based from the handbook of energy utilization

Table 2. Some characteristics of yam bean genotypes used in the experiment.

\begin{tabular}{|c|l|}
\hline ENTRY & \multicolumn{1}{|c|}{ DESCRIPTION } \\
\hline $\mathrm{G}_{1}$ & $\begin{array}{l}\text { Green-stalked, brown-colored roots with dark-brown surface color. Medium-sized, } \\
\text { monotuberous,semi-round, fairly lobed roots, dentate leaves, flowers light lavender borne } \\
\text { in racemes, medium maturing. }\end{array}$ \\
\hline $\mathrm{G}_{2}$ & $\begin{array}{l}\text { Green-stalked, light-brown and smooth, big root size line without any lobe, flowers are } \\
\text { borne alternately with lavender to white in color, big trifoliate leaves borne in just a short } \\
\text { vine, late maturing roots. }\end{array}$ \\
\hline $\mathrm{G}_{3}$ & $\begin{array}{l}\text { Red-stalked, light brown-colored roots, little bit bigger than } \mathrm{G}_{2} \text {; lobed roots with dentate } \\
\text { leaves, flowers light lavender in color; early maturing roots. }\end{array}$ \\
\hline $\mathrm{G}_{4}$ & $\begin{array}{l}\text { Dark-green stalk; roots small, a little bit pointed end with strigose hairs; deeply lobed, } \\
\text { dark-green leaves; long vines; flowers borne in clusters, deep lavender; medium to late } \\
\text { maturing roots. }\end{array}$ \\
\hline $\mathrm{G}_{5}$ & $\begin{array}{l}\text { Light-green stalk; medium-sized light-brown and smooth, round, monotuberous roots; } \\
\text { light lavender flowers borne in racemes. }\end{array}$ \\
\hline
\end{tabular}

G: Genotype by Pimentel (1980) and from relevant literatures of Mendoza (2007), Mendoza and Samson (2002), and Moerschner and Gerowitt (2000) as cited by Bockhari-Gevao, et al (2005) 
Table 3. Energy equivalents of labor, seed and root for yam bean production

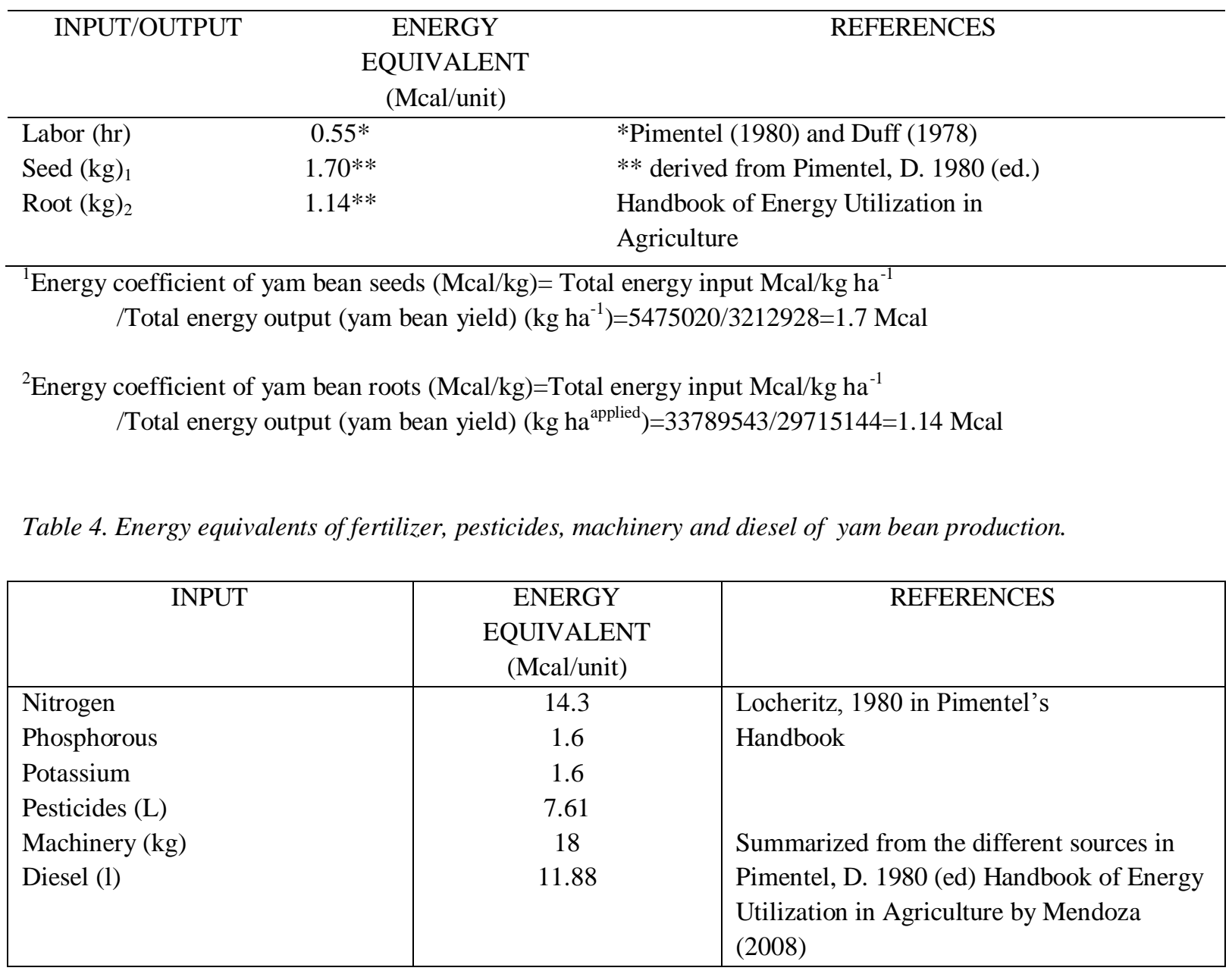

Energy input of machinery and direct diesel energy use of yam bean production are $180 \mathrm{Mcal} / \mathrm{ha}$ and 172.10 (L ha $^{-1}$, respectively (BASILIO, 2000).

Combined analysis from a series of split-plot experiments across the sites (five genotypes and four fertilizer levels) was used in this study. Analysis of variance (ANOVA) was used in the analysis of the data. SAS software four windows $(6.12 \mathrm{v})$ was used for the single site and combined analysis of the experiment. Treatments were compared using the Least Significant Difference (LSD) test at the 0.05 to 0.01 probability levels of significance.

\section{RESULTS AND DISCUSSION}

\section{Soil and Climatic Characteristics of the Sites}

\section{Soil Characteristics}

Site 1 ( Sarrat, Ilocos Norte). The site (Barangay Cabuloan) is $7 \mathrm{~km}$ east of Laoag City. It is bounded in the east by the town of Piddig, in the south by San Nicolas, west by Laoag City and north by Vintar. Aside, it is bounded in the north by a mountain traversing from east to west dividing Sarrat from the town of Vintar and south by Padsan river. The soil type used for the study was identified as Umingan clay loam with particle size distribution of $13.4 \%$ sand; $35.8 \%$ silt; and $50.8 \%$ clay. Based from the pre-planting soil analysis results conducted by the Bureau of Soils Laboratory-Ilocos Norte, the soil physico-chemical properties of the experimental sites are as follows: $\mathrm{pH}$ of $6.30 ; 1.34 \%$ organic matter $(\mathrm{OM})$ content; $0.67 \%(\mathrm{~N}) ; 36.08 \mathrm{ppm}(\mathrm{P})$; and $175.51 \mathrm{ppm} \mathrm{K})$. The soil in the experimental site is light textured and generally well-drained soil (Table 5). 
Site 2 (Dingras, Ilocos Norte). Dingras is located $18^{0} 6^{\prime} 33 \mathrm{~N}$ latitude and $120^{\circ} 41^{\prime} 34 \mathrm{E}$ longitude. It is $20 \mathrm{~km}$ from Laoag City. The study site (Barangay Madamba) is bounded by the towns of Piddig and Solsona in the north; by Nueva Erain the east; in the south by Marcos; and by Sarrat in the west. San Manuel silt loam was identified as the soil type of this site with particle size distribution of $20.4 \%$ sand; $58 \%$ silt; and $21.6 \%$ clay (Table 5).

Site 3 (Bangui, Ilocos Norte). The site is located in the far northern end of the province; bounded in the north by South China Sea; east by the towns of Pagudpud and Dumalneg; west by the town of Burgos; and south by the towns of Vintar. It lies between latitudes $18^{0} 25^{\prime}$ and $18^{0} 33^{\prime} \quad \mathrm{N}$ and longitudes $120^{\circ} 41^{\prime}$ and $120^{\circ} 50^{\prime} \mathrm{E}$. It is $64 \mathrm{~km}$ north of Laoag City. San Lorenzo (the study area) is the barangay at the heart of the town.

Bangui consists of mountainous lands which occupy $>50 \%$ of the total land area. The agricultural lands are predominantly planted to seasonal annual crops such as rice, corn, garlic, lowland vegetables and some legumes as cowpea, mungbean and yam bean. The soil type of this site was identified as San Fernando clay with particle size distribution of $28.2 \%$ sand; $35.6 \%$ silt and $36.2 \%$ clay (Table 5 ).

Table 5. Physico-chemical properties of the soils from the three sites before the experiment in Ilocos Norte, Philippines. 2010-2011.

\begin{tabular}{lccc}
\hline \hline \multicolumn{1}{c}{ Soil Property } & $\begin{array}{c}\text { Site 1 (Sarrat, Ilocos } \\
\text { Norte) }\end{array}$ & $\begin{array}{c}\text { Site 2 (Dingras, Ilocos } \\
\text { Norte) }\end{array}$ & $\begin{array}{c}\text { Site 3 (Bangui Ilocos } \\
\text { Norte) }\end{array}$ \\
\hline & & & \\
$\mathrm{pH}$ & 6.30 & 6.23 & 6.41 \\
Organic matter $(\%)$ & 1.34 & 0.90 & 0.72 \\
Nitrogen, N (\%) & 0.067 & 0.045 & 0.036 \\
Phosphorus,P(ppm) & 36.08 & 17.85 & 12.54 \\
Potassium, K (ppm) & 175.51 & 135.34 & 148.73 \\
Texture & Light & Light & Light \\
Particle size (\%) & & & \\
$\quad$ sand & 13.4 & 20.4 & 28.2 \\
$\quad$ silt & 35.8 & 58.0 & 35.6 \\
$\quad$ clay & 50.8 & 21.6 & 36.2 \\
\hline \hline
\end{tabular}

Source: Soil survey of Ilocos Norte, Philippines (Mangloñgat et al., 1980)

\section{Climatic Characteristics}

Generally, Ilocos Norte has a Type 1 climate based on the Corona Classification. Type 1 characterized by two pronounced seasons: dry and wet seasons. However, climatic variables (rainfall, temperature and windspeed) vary across the province (PAGASA, 1998). Data on monthly minimum, maximum and mean temperatures $\left({ }^{0} \mathrm{C}\right)$, rainfall $(\mathrm{mm})$, wind speed $\left(\mathrm{ms}^{-1}\right)$, sunshine $\left(\mathrm{mn}^{-1}\right)$ in the experimental area during the cropping season (field experimental period) are shown in Table 6 .

Site 1 (Sarrat, Ilocos Norte) . The average minimum and maximum temperatures ranged 14.3$23.4{ }^{\circ} \mathrm{C}$ and $33.2-38.0{ }^{\circ} \mathrm{C}$, respectively. Highest rainfall was recorded in July 2008 (1505 mm) and no precipitation was recorded in December 2009. Wind speed ranged 2-3 $\mathrm{ms}^{-1}$ and recorded sunshine ranged 422-647 $\mathrm{mn}^{-1}$. (PAGASA- Laoag City, 2009). There is a slight modification of the climate in this site since Sarrat is bounded by a mountain traversing from east to west.

Site 2 (Dingras, Ilocos Norte).Prevailing climatic factors are similar to Site 1, since Site 2 is adjacent to Site 1. However, the absence of mountain (features of topography) around the area makes it better for growing crops since the weather is fair as in Site 1 with two distinct dry and wet seasons. 
For Sites 1 and 2, the total amount of rainfall received during the cropping season was 2,997.9 $\mathrm{mm}$ with monthly average minimum and maximum temperatures of 20.36 and $35.24{ }^{\circ} \mathrm{C}$, respectively. The average prevailing wind in Sites 1 and 2 is $3.23 \mathrm{~m} \mathrm{~s}^{-1}$

Site 3 (Bangui, Ilocos Norte). The average monthly minimum, maximum and mean temperatures ranged $24.6-28.6^{\circ} \mathrm{C}$ and 26.2 to $29.9^{\circ} \mathrm{C}$, respectively. The highest rainfall was recorded in December with $1879 \mathrm{~mm}$. Prevailing wind speed recorded ranged 5.79 to $12.35 \mathrm{~ms}^{-1}$ with recorded average of $8.01 \mathrm{~m} \mathrm{~s}^{-1}$.

The total rainfall received during the cropping period was $3,230.78 \mathrm{~mm}$, with average monthly minimum and maximum temperatures of 26.44 and $28.03{ }^{\circ} \mathrm{C}$ respectively (NWPDC-BBWPP, 2009).

While yam bean favorably grow and have high yields at optimum temperatures of $24^{0} \mathrm{C}$ and a welldrained soil (Siemonsma and Piluek, 1993), the climatic factors during the cropping season for the experimental Sites 1 and 2 were within optimum temperature range but within upper limit. On the other hand, the minimum temperature in Site 3 was within the upper limit. In addition, the soil in Site 3 contains high proportion of sand $(28.2 \%)$ and windy condition with windspeed of $8.01 \mathrm{~ms}^{-1}$. This also contributed to the quick drying of the soil in this site, thus may affect the growth and development of the plants.

Table 6. Climatic data during the experimental period from March 2010 to March 2011. Sarrat, Dingras and Bangui, Ilocos Norte

Year/Month Temperature
Rainfall

(mm)

Minimum

\section{$\left({ }^{0} \mathrm{C}\right)$}

$(\mathrm{mm})$

Maximum
Prevailing Wind Sunshine

Speed

$\left(\mathrm{mn}^{-1}\right)$

\section{SITE 1 and 2*}

2008 MAR

APRIL

MAY

JUNE

JULY

AUG

SEPT

OCT

NOV
16.4

22.1

23.2

23.3

22.9

22.2

23.4

22.3

20.0
35.5

36.3

35.6

38.0

34.2

34.8

35.2

35.6

34.4 $\left(\mathrm{ms}^{-1}\right)$ 


$\begin{array}{rrrccc}\text { DEC. } & 16.0 & 34.2 & 0 & 3 & 500.6 \\ \text { 2009 JAN } & 14.3 & 33.6 & \text { Trace } & 3 & 464.8 \\ \text { FEB } & 17.5 & 35.0 & \text { Trace } & 2 & 510.0 \\ \text { MAR } & 21.1 & 35.7 & 0.1 & 3 & 602.1\end{array}$

SITE 3***

2008 MAR

APRIL

MAY

JUNE

JULY

AUG

SEPT

OCT

NOV

DEC

2009 JAN

FEB

MAR
27.9

26.22

26.54

28.59

24.78

25.01

27.56

27.20

25.66

25.94

24.58

26.01

27.79
28.05

27.46

28.78

29.9

26.67

26.89

28.76

29.76

26.90

28.18

26.17

27.52

29.38
0

6.89

No record

\section{* Source: PAGASA, Laoag International Airport,
**Source: North Wind Power Development Corp
Days to Germination, Flowering and Maturity
of Yam Bean Genotypes}

Site 1 (Sarrat, Ilocos Norte). Days to germination in yam bean plants was significantly affected by fertilizer treatment in Site 1 (Table 7). Days to germination in this Site was observed at 4-6 days.
Seeds applied with organic fertilizer germinated earlier (4 DAP), followed by the control plants (5 DAP) and the latest to germinate was by those plants applied with inorganic fertilizer. Genotype did not significantly affect days to germination of the yam bean plants. However, Genotypes 1, 2 and 
4 germinated 5 DAP while Genotype 3 and Genotype 5 germinated at 6 DAP.

Days to flowering was not significantly affected by fertilizer treatments (Table 7). However, applied with inorganic fertilizer as well as control plants flowered earlier than the other fertilizer treatments. Days to flowering differed with genotypes. Among the genotypes, Genotype 4 flowered the latest $(82$ DAP) as compared with the other genotypes that flowered 78 (Genotypes 2 and 3) and 79 (Genotypes 1 and 5) DAP.

Fertilizer treatments significantly affected days to maturity. Plants matured the earliest in control treatments, while plants applied with organic fertilizer matured the latest (97 DAP). Among the genotypes, Genotype 3 was the earliest to mature (92 DAP), while the latest to mature was Genotype 4 (99 DAP).

In Site 1, OF application was found out to shorten germination, flowering and maturity. The addition of OF had probably contributed to the improvement of the soil since it is high in clay (Table 5). The porosity has been improved and thus contributed to proper drainage in the soil, in addition to its OM content as source of additional nutrients for proper growth of the plants. For the genotype that flowered the earliest across fertilizer treatments, this could be a genotype characteristics being an early maturing type (98 DAP).

Site 2 (Dingras, Ilocos Norte). Fertilizer treatments significantly affected days to germination of the yam bean plants (Table 7). The plants germinated 4-5 days after planting in plants applied with $50 \% \mathrm{OF}+50 \% \mathrm{IF}$, the earliest to germinate. With regards to genotypes, days to germination was not significantly affected although Genotype 4 and Genotype 5 germinated the earliest (4 DAP), which could be a genotype characteristics.

Flowering was significantly affected by both fertilizer treatments and genotypes. 50\% OF + $50 \%$ IF application resulted to earliest flowering of the plants with 76 DAP, while the rest of the treatments flowered at the same time (80 DAP). Among the genotypes, Genotype 3 flowered the earliest with 75 DAP while Genotype 5 was the latest to flower (82 DAP)
The days to maturity was significantly affected by both fertilizer ad genotypes. Control plants matured the earliest while those with OF matured the latest with 102 DAP. Among the genotypes, Genotype 3 matured the earliest with 97 DAP and the latest was Genotype 4.

In Site 2, the soil and climatic characteristics favored the performance of the genotypes. That the particle size distribution of sand, silt and clay (20.4, 58 and $21.6 \%$ respectively) is just balanced. Such that the use of $50 \% \mathrm{OF}+50 \% \mathrm{IF}$ is the best for this type of site with sufficient amount of soil OM that favored the activity of microorganisms and formation of soil aggregates which improved the soil structure favorable for crop growth and development.

Site 3 (Bangui, Ilocos Norte). Days to germination was not significantly affected by fertilizer. The yam bean plants germinated longer (by 3-4 days) as compared to Sites 1 and 2. Genotypes on the other hand significantly affected days to germination. Genotype 1 was the earliest to germinate (8 DAP) while Genotype 2 the latest with 10 DAP.

Fertilizer treatments did not affect days to flowering of yam bean plants in Site 3. Control plants flowered the earliest (82 DAP), while it differed with genotype wherein Genotype 1 flowered the earliest in this site. 
Table 7. Days to germination, flowering and maturity of yam bean genotypes grown with different fertilizer treatments at three sites in Ilocos Norte, Philippines. 2010-2011 Cropping season

\begin{tabular}{|c|c|c|c|c|c|c|c|c|c|c|c|c|c|c|c|}
\hline \multirow[b]{3}{*}{$\begin{array}{c}\text { GENOTYP } \\
\text { E }\end{array}$} & \multicolumn{15}{|c|}{ Days to Germination } \\
\hline & \multicolumn{5}{|c|}{ Site 1 (Sarrat, Ilocos Norte) } & \multicolumn{5}{|c|}{ Site 2 (Dingras, Ilocos Norte) } & \multicolumn{5}{|c|}{ Site 3 (Bangui, Ilocos Norte) } \\
\hline & $\mathrm{C}$ & OF & $\begin{array}{c}50 \% \mathrm{OF}+ \\
50 \% \mathrm{IF}\end{array}$ & IF & Mean & $\mathbf{C}$ & $\mathrm{OF}$ & $\begin{array}{l}50 \% \mathrm{OF} \\
+50 \% \mathrm{IF}\end{array}$ & IF & Mean & $\mathrm{C}$ & OF & $\begin{array}{l}50 \% \mathrm{OF} \\
+50 \% \mathrm{IF}\end{array}$ & IF & Mean \\
\hline 1 & 5 & 5 & 6 & 5 & 5 & 6 & 6 & 4 & 7 & $6 \mathbf{a}$ & 8 & 8 & 8 & 9 & $8 \mathrm{c}$ \\
\hline 2 & 5 & 3 & 6 & 6 & 5 & 5 & 6 & 4 & 5 & $5 \mathbf{a b}$ & 9 & 10 & 10 & 10 & $10 \mathbf{a}$ \\
\hline 3 & 4 & 6 & 7 & 6 & 6 & 4 & 7 & 4 & 6 & $5 \mathbf{a b}$ & 8 & $\mathbf{1 0}$ & 9 & 9 & $9 \mathrm{~b}$ \\
\hline 4 & 4 & 4 & 5 & 5 & 5 & 5 & 3 & 4 & 5 & $4 \mathrm{~b}$ & 10 & 8 & 10 & 10 & $9 \mathrm{~b}$ \\
\hline 5 & 6 & 4 & 7 & 7 & 6 & 5 & 5 & 4 & 3 & $4 \mathrm{~b}$ & 9 & 9 & 9 & 10 & $9 \mathrm{~b}$ \\
\hline \multirow[t]{4}{*}{ MEAN } & 5 bc & $4 \mathrm{c}$ & $6 \mathbf{a}$ & $6 \mathbf{a b}$ & & 5 a & 5 a & $4 \mathrm{~b}$ & $5 \mathbf{a}$ & & 9 & 9 & 9 & 9 & \\
\hline & \multicolumn{5}{|c|}{$\mathrm{F}: \operatorname{Pr}>\mathrm{F}=0.0018 ; \mathrm{LSD}=0.96$} & \multicolumn{5}{|c|}{$\mathrm{F}: \operatorname{Pr}>\mathrm{F}=0.0277 ; \mathrm{LSD}=1.0$} & \multicolumn{5}{|c|}{$\mathrm{F}: \operatorname{Pr}>\mathrm{F}=0.3151 ; \mathrm{LSD}=0.82$} \\
\hline & \multicolumn{5}{|c|}{$\mathrm{G}: \operatorname{Pr}>\mathrm{F}=0.0617 ; \mathrm{LSD}=1.07$} & \multicolumn{5}{|c|}{$\mathrm{G}: \operatorname{Pr}>\mathrm{F}=0.1057 ; \mathrm{LSD}=1.24$} & \multicolumn{5}{|c|}{ G: $\operatorname{Pr}>F=0.0322 ; L S D=0.92$} \\
\hline & \multicolumn{5}{|c|}{ F X G: $\operatorname{Pr}>F=0.4445$} & \multicolumn{5}{|c|}{ F X G: $P r>F=0.3190$} & \multicolumn{5}{|c|}{ F X G: $\operatorname{Pr}>F=0.4774$} \\
\hline \multicolumn{16}{|c|}{ Days to Flowering } \\
\hline 1 & 79 & 82 & 75 & 78 & $79 a b$ & 84 & 84 & 80 & 80 & $82 \mathrm{a}$ & 78 & 83 & 84 & 80 & $81 \mathrm{~b}$ \\
\hline 2 & 77 & 80 & 82 & 73 & $78 \mathrm{~b}$ & 80 & 80 & 77 & 82 & $80 \mathrm{ab}$ & 84 & 84 & 84 & 83 & $84 \mathrm{a}$ \\
\hline 3 & 73 & 78 & 83 & 77 & $78 \mathrm{~b}$ & 76 & 77 & 74 & 74 & $75 \mathrm{c}$ & 84 & 84 & 84 & 83 & $84 \mathrm{a}$ \\
\hline 4 & 83 & 81 & 84 & 82 & $82 \mathrm{a}$ & 82 & 82 & 80 & 81 & $81 \mathrm{a}$ & 82 & 84 & 84 & 84 & $84 \mathrm{a}$ \\
\hline 5 & 77 & 82 & 77 & 82 & $79 \mathrm{ab}$ & 80 & 77 & 71 & 82 & $77 \mathrm{c}$ & 84 & 85 & 84 & 84 & $84 \mathrm{a}$ \\
\hline \multirow[t]{4}{*}{ Mean } & 78 & 80 & 80 & 78 & & $80 \mathrm{a}$ & $80 \mathrm{a}$ & $76 \mathrm{~b}$ & $80 \mathrm{a}$ & & $82 \mathrm{~b}$ & $84 \mathrm{a}$ & $84 \mathrm{a}$ & $83 \mathrm{ab}$ & \\
\hline & \multicolumn{5}{|c|}{$\mathrm{F}: \mathrm{Pr}>\mathrm{F}=0.2273 ; \mathrm{LSD}=1.85$} & \multicolumn{5}{|c|}{$\mathrm{F}: \mathrm{Pr}>\mathrm{F}=0.0124 ; \mathrm{LSD}=2.65$} & \multicolumn{5}{|c|}{$\mathrm{F}: \operatorname{Pr}>\mathrm{F}=0.0732 ; \mathrm{LSD}=1.57$} \\
\hline & \multicolumn{5}{|c|}{ G: $\operatorname{Pr}>F=0.0607 ; \mathrm{LSD}=3.45$} & \multicolumn{5}{|c|}{$\mathrm{G}: \mathrm{Pr}>\mathrm{F}=0.0033 ; \mathrm{LSD}=2.96$} & \multicolumn{5}{|c|}{ G: $\operatorname{Pr}>F=0.0067 ; \mathrm{LSD}=1.75$} \\
\hline & \multicolumn{5}{|c|}{ F x G: $\operatorname{Pr}>F=0.0629$} & \multicolumn{5}{|c|}{ F X G: $P r>F=0.4163$} & \multicolumn{5}{|c|}{ F X G: $\operatorname{Pr}>F=0.6541$} \\
\hline
\end{tabular}


Jocelyn A. Bernabe / Energy utilization of yam bean....

\begin{tabular}{|c|c|c|c|c|c|c|c|c|c|c|c|c|c|c|c|}
\hline \multicolumn{16}{|c|}{ Days to Maturity } \\
\hline 1 & 95 & 98 & 97 & 97 & $97 \mathrm{~b}$ & 100 & 103 & 102 & 102 & $102 \mathrm{ab}$ & 119 & 119 & 119 & 120 & $119 \mathrm{~b}$ \\
\hline 2 & 94 & 97 & 95 & 94 & $95 \mathrm{bc}$ & 99 & 102 & 100 & 99 & $100 \mathrm{bc}$ & 121 & 121 & 121 & 121 & $121 \mathrm{a}$ \\
\hline 3 & 92 & 93 & 92 & 93 & $92 \mathrm{~d}$ & 97 & 98 & 97 & 98 & $97 \mathrm{~d}$ & 119 & 121 & 120 & 120 & $120 \mathrm{ab}$ \\
\hline 4 & 97 & 103 & 97 & 98 & $99 \mathrm{a}$ & 102 & 108 & 102 & 103 & $104 \mathrm{a}$ & 121 & 119 & 122 & 122 & $121 \mathrm{a}$ \\
\hline 5 & 92 & 95 & 95 & 93 & $94 \mathrm{~cd}$ & 97 & 100 & 101 & 98 & $99 \mathrm{~cd}$ & 120 & 120 & 121 & 121 & $121 \mathrm{ab}$ \\
\hline \multirow[t]{4}{*}{ Mean } & $94 \mathrm{~b}$ & $97 \mathrm{a}$ & $95 \mathrm{~b}$ & $95 \mathrm{~b}$ & & $99 \mathrm{bc}$ & $102 \mathrm{a}$ & $100 \mathrm{~b}$ & $100 \mathrm{~b}$ & & 120 & 120 & 121 & 121 & \\
\hline & \multicolumn{5}{|c|}{$\mathrm{F}: \operatorname{Pr}>\mathrm{F}=0.0114 ; \mathrm{LSD}=1.85$} & \multicolumn{5}{|c|}{$\mathrm{F}: \operatorname{Pr}>\mathrm{F}=0.0170 ; \mathrm{LSD}=1.93$} & \multicolumn{5}{|c|}{$\mathrm{F}: \operatorname{Pr}>\mathrm{F}=0.3179 ; \mathrm{LSD}=1.15$} \\
\hline & \multicolumn{5}{|c|}{ G: $\operatorname{Pr}>F=0.0001 ; \mathrm{LSD}=2.07$} & \multicolumn{5}{|c|}{$\mathrm{G}: \operatorname{Pr}>\mathrm{F}=0.0481 ; \mathrm{LSD}=2.16$} & \multicolumn{5}{|c|}{$\mathrm{G}: \operatorname{Pr}>\mathrm{F}=0.0481 ; \mathrm{LSD}=1.28$} \\
\hline & \multicolumn{5}{|c|}{ F X G: $\operatorname{Pr}>F=0.7767$} & \multicolumn{5}{|c|}{ F X G: $P r>F=0.7221$} & \multicolumn{5}{|c|}{ F X G: $\operatorname{Pr}>F=0.5001$} \\
\hline
\end{tabular}


Table 8. Yield $\left(\mathrm{t} \mathrm{ha} \mathrm{a}^{-1}\right)$ and harvest index of yam bean genotypes grown with different fertilizer treatments at three sites in IlocosNorte. 2010-2011 Cropping Season

Within a column ( $\mathrm{G}$ means) and/or within a row (F means), means followed by different letters are significantly different at $5 \%$ level of significance by LS

\begin{tabular}{|c|c|c|c|c|c|c|c|c|c|c|c|c|c|c|c|}
\hline \multirow[b]{3}{*}{$\begin{array}{c}\text { GENOTYP } \\
\text { E }\end{array}$} & \multicolumn{15}{|c|}{ Harvest Index } \\
\hline & \multicolumn{5}{|c|}{ Site 1 (Sarrat, Ilocos Norte) } & \multicolumn{5}{|c|}{ Site 2 (Dingras, Ilocos Norte) } & \multicolumn{5}{|c|}{ Site 3 (Bangui, Ilocos Norte) } \\
\hline & $\mathbf{C}$ & $\mathbf{O F}$ & $\begin{array}{l}\mathbf{5 0 \%} \text { OF } \\
+\mathbf{5 0 \%} \text { IF }\end{array}$ & IF & Mean & $\mathbf{C}$ & OF & $\begin{array}{c}50 \% \text { OF } \\
+50 \% \text { IF }\end{array}$ & IF & Mean & $\mathbf{C}$ & OF & $\begin{array}{c}50 \% \text { OF } \\
+50 \% \text { IF }\end{array}$ & IF & Mean \\
\hline 1 & $\mathbf{0 . 8 0}$ & 0.79 & 0.82 & 0.78 & $0.80 \mathrm{~b}$ & $\mathbf{0 . 8 3}$ & 0.84 & 0.84 & $\mathbf{0 . 8 3}$ & $\mathbf{0 . 8 3}$ & 0.76 & $\mathbf{0 . 7 3}$ & 0.78 & 0.72 & 0.72 \\
\hline 2 & $\mathbf{0 . 8 1}$ & $\mathbf{0 , 8 0}$ & $\mathbf{0 . 8 1}$ & $\mathbf{0 . 7 8}$ & $0.80 \mathrm{~b}$ & $\mathbf{0 . 8 8}$ & $\mathbf{0 . 8 8}$ & 0.79 & 0.86 & 0.85 & $\mathbf{0 . 7 8}$ & 0.74 & 0.73 & $\mathbf{0 . 7 2}$ & $\mathbf{0 . 8 0}$ \\
\hline 3 & 0.80 & $\mathbf{0 . 8 0}$ & 0.84 & 0.78 & $0.81 \mathrm{~b}$ & $\mathbf{0 . 8 5}$ & 0.87 & 0.80 & 0.85 & 0.84 & 0.77 & 0.72 & 0.74 & $\mathbf{0 . 7 0}$ & 0.79 \\
\hline 4 & 0.84 & $\mathbf{0 . 8 3}$ & 0.85 & 0.80 & 0.83 a & 0.85 & 0.86 & 0.88 & $\mathbf{0 . 8 3}$ & 0.86 & 0.73 & 0.73 & 0.76 & 0.72 & 0.81 \\
\hline 5 & $\mathbf{0 . 8 1}$ & $\mathbf{0 . 8 0}$ & 0.85 & $\mathbf{0 . 8 4}$ & 0.83 a & $\mathbf{0 . 8 3}$ & 0.87 & 0.81 & 0.87 & $\mathbf{0 . 8 4}$ & 0.76 & $\mathbf{0 . 7 5}$ & $\mathbf{0 . 7 2}$ & 0.73 & $\mathbf{0 . 8 0}$ \\
\hline \multirow[t]{4}{*}{ Mean } & $\begin{array}{l}0.81 \\
b\end{array}$ & $\begin{array}{l}\text { 0.80 } \\
\text { bc }\end{array}$ & $0.83 \mathrm{a}$ & $\begin{array}{l}0.78 \\
\text { c }\end{array}$ & & $\begin{array}{l}0.85 \\
\text { ab }\end{array}$ & 0.86 a & $0.83 \mathrm{ab}$ & $0.85 \mathrm{ab}$ & & $\begin{array}{l}0.76 \\
\mathrm{a}\end{array}$ & $\begin{array}{l}0.73 \\
\text { ab }\end{array}$ & $0.74 \mathrm{ab}$ & $0.72 \mathrm{~b}$ & \\
\hline & \multicolumn{5}{|c|}{ F: $\operatorname{Pr}>$ F $=0.002 ;$ LSD $=0.02$} & \multicolumn{5}{|c|}{$F: \operatorname{Pr}>F=0.001 ;$ LSD fert $=0.02$} & \multicolumn{5}{|c|}{$F: \operatorname{Pr}>F=0.00021 ;$ LSD fert $=0.03$} \\
\hline & \multicolumn{5}{|c|}{$G: \operatorname{Pr}>F=0.0032 ;$ LSD $=0.02$} & \multicolumn{5}{|c|}{ G: $\operatorname{Pr}>\mathrm{F}=0.1123 ;$ LSD gen $=0.02$} & \multicolumn{5}{|c|}{ G: $\operatorname{Pr}>F=0.2134 ;$ LSD gen $=0.04$} \\
\hline & \multicolumn{5}{|c|}{ F x G : Pr > F = 0.1899 ns } & \multicolumn{5}{|c|}{ F x G: $\operatorname{Pr}>F=0.1234 n s$} & \multicolumn{5}{|c|}{ F $x$ G: $\operatorname{Pr}>F=0.3212 n s$} \\
\hline \multicolumn{16}{|c|}{$\begin{array}{l}\text { Yield }\left(\mathrm{t} \mathrm{ha}^{-1}\right) \\
\end{array}$} \\
\hline 1 & 50.67 & 63.33 & 70.33 & 49.67 & $\mathbf{5 8 . 5 0}$ & $\mathbf{5 7 . 8 3}$ & 60.00 & 66.50 & 62.50 & 61.71 & 51.67 & 49.50 & 33.67 & 44.00 & $\begin{array}{c}44.71 \\
\mathrm{a}\end{array}$ \\
\hline 2 & 58.33 & 61.13 & 58.83 & 57.67 & 58.99 & 63.00 & 55.00 & 66.17 & 59.00 & 60.79 & 54.00 & 45.33 & 41.33 & 42.17 & $\begin{array}{c}45.71 \\
a\end{array}$ \\
\hline 3 & 61.33 & 55.00 & 65.50 & 50.33 & 58.04 & 59.00 & 56.83 & 63.67 & 54.50 & 58.50 & 46.33 & 56.70 & 43.17 & 41.17 & $\begin{array}{c}46.84 \\
a\end{array}$ \\
\hline 4 & 47.50 & 57.17 & 62.17 & 58.67 & 56.38 & 51.00 & $\mathbf{5 9 . 5 0}$ & 59.17 & 58.17 & 56.96 & 47.50 & 56.17 & 47.67 & 39.50 & $\begin{array}{c}47.71 \\
\mathbf{a}\end{array}$ \\
\hline 5 & 63.67 & 55.33 & 57.67 & 39.73 & 54.10 & 58.00 & 55.00 & 73.50 & 72.17 & 64.67 & 46.33 & 57.17 & 49.67 & 35.33 & $\begin{array}{c}47.13 \\
\mathrm{a}\end{array}$ \\
\hline Mean & $\begin{array}{c}56.3 \\
\text { bc }\end{array}$ & $\begin{array}{c}58.39 \\
\text { ab }\end{array}$ & $62.90 \mathrm{a}$ & $\begin{array}{c}51.21 \\
\text { c }\end{array}$ & & $\begin{array}{c}57.77 \\
\text { b }\end{array}$ & $\begin{array}{c}57.27 \\
\text { b }\end{array}$ & $65.80 \mathrm{a}$ & $\begin{array}{c}61.27 \\
\text { ab } \\
\end{array}$ & & $\begin{array}{c}49.17 \\
\text { ab }\end{array}$ & $\begin{array}{c}\mathbf{5 2 . 9 7} \\
\mathbf{a}\end{array}$ & 43.10 bc & $40.43 \mathrm{c}$ & \\
\hline & \multicolumn{5}{|c|}{$F: \operatorname{Pr}>F=0.0097 ; L S D=6.59$} & \multicolumn{5}{|c|}{$F: \operatorname{Pr}>F=0.0441 ;$ LSD $=6.54$} & \multicolumn{5}{|c|}{ F: $\operatorname{Pr}>\mathrm{F}=0.0017 ; \mathrm{LSD}=6.52$} \\
\hline & \multicolumn{5}{|c|}{ G: $\operatorname{Pr}>F=0.6603 ;$ LSD $=7.37$} & \multicolumn{5}{|c|}{ GP: $\operatorname{Pr}>\mathrm{F}=0.2637 ; \mathrm{LSD}=7.31$} & \multicolumn{5}{|c|}{ G: $\operatorname{Pr}>\mathrm{F}=0.9223 ; \mathrm{LSD}=7.29$} \\
\hline & \multicolumn{5}{|c|}{ F X G : Pr $>$ F = 0.1235 } & \multicolumn{5}{|c|}{ F X G: $\operatorname{Pr}>F=0.6532$} & \multicolumn{5}{|c|}{ F X G: Pr >F =0.3972 } \\
\hline
\end{tabular}




\section{Harvest Index}

Site 1 (Sarrat, Ilocos Norte). Results showed that harvest index (HI) was significantly affected by fertilizer treatments, while genotypes varied significantly, while there are no interaction effects observed (Table 8). The proportion of economic yield to that of biological yield was observed to be highest in plants applied with $50 \% \mathrm{OF}+50 \% \mathrm{IF}$ ( $0.83 \mathrm{~kg} \mathrm{ha}^{-1}$ ) and the lowest was the plants applied with inorganic fertilizer $\left(0.78 \mathrm{~kg} \mathrm{ha}^{-1}\right)$. Genotype 4 and Genotype 5 were observed to be the highest in harvest index in this site with $0.83 \mathrm{~kg} \mathrm{ha}^{-1}$.

Site 2 (Dingras, Ilocos Norte).HI was significantly affected by fertilizer treatments but not with genotypes, while no interaction effects was observed. In this site, the plants applied with organic fertilizer had the highest HI value $(0.86 \mathrm{~kg}$ $\left.\mathrm{ha}^{-1}\right)$. The lowest was obtained from plants applied with $50 \% \mathrm{OF}+50 \% \mathrm{IF}$ with $0.83 \mathrm{~kg} \mathrm{ha}^{-1}$. Among the genotypes, the highest $\mathrm{HI}$ was obtained by Genotype 4 with $0.86 \mathrm{~kg} \mathrm{ha}^{-1}$ in this particular site.

Site 3(Bangui, Ilocos Norte). Harvest index in this sitewas significantly affected by fertilizer treatments but did not vary with genotypes. Unfertilized plants obtained the highest HI among the treatments used with $0.76 \mathrm{~kg} \mathrm{ha}^{-1}$, while the lowest was from plants applied with inorganic fertilizer. Among the genotypes, Genotype 4 had the highest $\mathrm{HI}\left(0.81 \mathrm{~kg} \mathrm{ha}^{-1}\right)$, while the lowest was obtained from Genotype 1.

There was no significant interaction effects on the fertilizer, genotype and site. However, HI varied across sites wherein, site was found out to be significant against the other sites with respect to HI. Site 2 produced the highest HI, followed by Site 1, and the lowest was Site 3. This suggest a better partitioning of dry matter to yield in Site 2 .

\section{Yield of Yam Bean Genotypes}

Site 1 ( Sarrat, Ilocos Norte). Yam bean yield was significantly affected by fertilizer but not by genotype in this site (Table 14). The application of $50 \% \mathrm{OF}+50 \% \mathrm{IF}$ to the plants produced the highest yield $\left(62.90 \mathrm{t} \mathrm{ha}^{-1}\right)$ followed by those applied with organic fertilizer $\left(58.39 \mathrm{t} \mathrm{ha}^{-1}\right)$, the lowest was with inorganic fertilizer $\left(51.21 \mathrm{t} \mathrm{ha}^{-1}\right)$. The genotypes have comparable yields, although
Genotype 2 produced the relatively highest yield ( $58.99 \mathrm{t} \mathrm{ha}^{-1}$ ) in this site.

Soil in this site is of Umingan type, where balanced proportions of soil particles of sand, silt and clay, an application of $50 \%$ OF $+50 \%$ IF was found to be favorable. It has a well established effect of organic fertilizer on texture, nutrient/water retention and microbial growth. Combination of $\mathrm{OF}$ and IF show immediate availability of fertilizer on variable growth stages. Organic fertilizer provides micronutrients and the other growth factors not normally supplied by inorganic fertilizers (Jones and Wild, 1975).

Site 2 (Dingras, Ilocos Norte). Similar trend results as in Site 1 were obtained. Yield was significantly affected by fertilizer but did not vary by genotypes. No interaction effects of fertilizer treatments by genotype. Highest yield was obtained in plots with $50 \%$ OF $+50 \%$ IF $\left(65.80 \mathrm{t} \mathrm{ha}^{-1}\right)$. With regards to genotype, Genotype 5 had the highest (64.67 $\mathrm{t} \mathrm{ha}^{-1}$ ) in this site.

With San Manuel silt loam type of soil in this site, it has high silt particle which is favorable for root crops, while this soil is low in available $\mathrm{N}$ as well as OM, using a combination of organic and inorganic fertilizer worked since $\mathrm{OF}$ is needed for the increase in microbial population for the biological $\mathrm{N}$ fixing activity of the plant as well as for the improvement of the soil properties and supply of other important nutrients not supplied by inorganic fertilizer.

Site 3 (Bangui, Ilocos Norte).Fertilizer treatments significantly affected the yield in this site (Table 8). Plants applied with organic fertilizer alone was observed to have obtained the highest yield $(52.97 \mathrm{t}$ $\mathrm{ha}^{-1}$ ), followed by control plants $49.17 \mathrm{t} \mathrm{ha}^{-1}$ while the lowest was from inorganic fertilizer applied plants (40.43 $\mathrm{t}$ ha ${ }^{-1}$. Yield did vary across genotypes, although Genotype 4 and 5 appeared to be adapted to the site and condition. Similarly, genotype by fertilizer interaction was not observed on yam bean yields.

Organic fertilizer worked well in Site 3. Based from the pre-soil analysis, this site is high in sand proportion, and has low OM, N and P. In addition, Site 3 has relatively strong windspeed $\left(8.1 \mathrm{~ms}^{-1}\right.$ vs. $2-3 \mathrm{~ms}^{-1}$ for Sites 1 and 2 ), thus the area easily dries 
up because it cannot retain moisture longer due to dominant particle size as well as prevailing strong winds in the area. Based on these results, the application of organic fertilizer alone increased the yield in this site. While the improved soil structure, nutrient/water retention and microbial activity had been attributed to $\mathrm{OF}$.

Among the sites, the highest average yield was obtained in Site 2 followed by Site 1 and the lowest was obtained in Site 3. Among the sites, significant difference in yield was observed. However, the interaction effects of fertilizer treatment and genotype was not observed. But, with fertilizer and genotype, there were no significant interaction effects. Based on the results, yield was significantly increased by the application of $50 \%$ OF $+50 \%$ IF, specifically for Sites 1 and 2, while organic fertilizer application alone was effective in Site 3 which is more prone to water deficit conditions 
Table 9. Summary table of cost and return analysis of yam bean genotypes grown with four fertilizer treatments in three sites of Ilocos Norte Philippines. 2010-2011 Cropping Season.

\begin{tabular}{|c|c|c|c|c|c|c|c|c|c|c|c|c|c|c|c|}
\hline \multirow[b]{2}{*}{$\begin{array}{l}\mathrm{G} \\
\text { en } \\
\text { o } \\
\text { ty } \\
\text { pe }\end{array}$} & \multicolumn{5}{|c|}{ Site 1 (Sarrat, Ilocos Norte) } & \multicolumn{5}{|c|}{ Site 2 (Dingras, Ilocos Norte) } & \multicolumn{5}{|c|}{ Site 3 (Bangui, Ilocos Norte) } \\
\hline & $\begin{array}{l}\text { Con } \\
\text { trol }\end{array}$ & $\mathbf{O F}$ & $\begin{array}{c}\mathbf{5 0} \\
\% \mathbf{O} \\
\mathbf{F}+ \\
\mathbf{5 0} \\
\% \mathbf{I} \\
\mathbf{F}\end{array}$ & IF & $\begin{array}{c}\text { Mea } \\
\mathbf{n}\end{array}$ & $\begin{array}{l}\text { Con } \\
\text { trol }\end{array}$ & $\mathbf{O F}$ & $\begin{array}{c}\mathbf{5 0} \\
\% \mathbf{O} \\
\mathbf{F}+ \\
\mathbf{5 0} \\
\% \mathbf{I} \\
\mathrm{F} \\
\end{array}$ & IF & $\begin{array}{c}\text { Mea } \\
\text { n }\end{array}$ & $\begin{array}{l}\text { Con } \\
\text { trol }\end{array}$ & $\mathbf{O F}$ & $\begin{array}{c}\mathbf{5 0} \\
\% \mathbf{O} \\
\mathbf{F}+ \\
\mathbf{5 0} \\
\% \mathbf{I} \\
\mathrm{F}\end{array}$ & IF & $\begin{array}{c}\text { Mea } \\
\text { n }\end{array}$ \\
\hline \multicolumn{16}{|c|}{ Total Production Cost $\left(\mathrm{PhP} \mathrm{ha}^{-1}\right)$} \\
\hline \multirow[t]{2}{*}{1} & 476 & 526 & 564 & 602 & 5423 & 468 & 518 & 556 & 595 & 5348 & 613 & 663 & 701 & 740 & 6798 \\
\hline & 10 & 10 & 40 & 88.2 & 7.05 & 60 & 60 & 90 & 38.2 & 7.05 & 60 & 60 & 90 & 38.2 & 7.05 \\
\hline \multirow[t]{2}{*}{2} & 472 & 522 & 561 & 599 & 5390 & 465 & 515 & 553 & 592 & 5315 & 610 & 660 & 698 & 737 & 6765 \\
\hline & 74 & 74 & 04 & 52.2 & 1.05 & 24 & 24 & 54 & 02.2 & 1.05 & 24 & 24 & 54 & 02.2 & 1.05 \\
\hline \multirow[t]{2}{*}{3} & 484 & 534 & 572 & 611 & 5507 & 477 & 527 & 565 & 603 & 5432 & 622 & 672 & 710 & 748 & 6882 \\
\hline & 50 & 50 & 80 & 28.2 & 7.05 & 00 & 00 & 30 & 78.2 & 7.05 & 00 & 00 & 30 & 78.2 & 7.05 \\
\hline \multirow[t]{2}{*}{4} & 478 & 528 & 566 & 605 & 5447 & 471 & 521 & 559 & 597 & 5372 & 616 & 666 & 704 & 742 & 6822 \\
\hline & 50 & 50 & 80 & 28.2 & 7.05 & 00 & 00 & 30 & 78.2 & 7.05 & 00 & 00 & 30 & 78.2 & 7.05 \\
\hline \multirow[t]{2}{*}{5} & 472 & 522 & 561 & 599 & 5390 & 472 & 522 & 561 & 599 & 5390 & 610 & 660 & 698 & 737 & 6765 \\
\hline & 74 & 74 & 04 & 52.2 & 1.05 & 74 & 74 & 04 & 52.2 & 1.05 & 24 & 24 & 54 & 02.2 & 1.05 \\
\hline $\mathrm{M}$ & 476 & 526 & 565 & 603 & 5431 & 470 & 520 & 559 & 597 & 5371 & 614 & 664 & 702 & 741 & 6806 \\
\hline ea & 91.6 & 91.6 & 21.6 & 69.8 & 8.65 & 91.6 & 91.6 & 21.6 & 69.8 & 8.65 & 41.6 & 41.6 & 71.6 & 19.8 & 8.65 \\
\hline $\mathrm{n}$ & & & & & & & & & & & & & & & \\
\hline \multicolumn{16}{|c|}{ Gross Income (PhP ha $\left.{ }^{-1}\right)$} \\
\hline \multirow[t]{2}{*}{1} & 253 & 316 & 351 & 248 & 2925 & 289 & 300 & 332 & 312 & 3085 & 258 & 247 & 168 & 220 & 2235 \\
\hline & 350 & 650 & 650 & 350 & 00 & 150 & 000 & 500 & 500 & 37.5 & 350 & 500 & 350 & 000 & 50 \\
\hline \multirow[t]{2}{*}{2} & 291 & 305 & 294 & 288 & 2949 & 315 & 275 & 330 & 295 & 3039 & 270 & 226 & 206 & 210 & 2285 \\
\hline & 650 & 650 & 150 & 350 & 50 & 000 & 000 & 850 & 000 & 62.5 & 000 & 650 & 650 & 850 & 37.5 \\
\hline \multirow[t]{2}{*}{3} & 306 & 275 & 327 & 251 & 2902 & 295 & 284 & 318 & 272 & 2925 & 231 & 283 & 215 & 205 & 2342 \\
\hline & 650 & 000 & 500 & 650 & 00 & 000 & 150 & 350 & 500 & 00 & 650 & 500 & 850 & 850 & 12.5 \\
\hline \multirow[t]{2}{*}{4} & 237 & 285 & 310 & 293 & 2818 & 255 & 297 & 295 & 290 & 2848 & 237 & 280 & 238 & 197 & 2385 \\
\hline & 500 & 850 & 850 & 350 & 87.5 & 000 & 500 & 850 & 850 & 00 & 500 & 850 & 350 & 500 & 50 \\
\hline \multirow[t]{2}{*}{5} & 318 & 276 & 288 & 198 & 2705 & 290 & 275 & 367 & 360 & 3233 & 231 & 285 & 248 & 176 & 2356 \\
\hline & 350 & 650 & 350 & 650 & 00 & 000 & 000 & 500 & 850 & 37.5 & 650 & 850 & 350 & 650 & 25 \\
\hline $\mathrm{M}$ & 281 & 291 & 314 & 256 & 2860 & 288 & 286 & 329 & 306 & 3026 & 245 & 264 & 215 & 202 & 2320 \\
\hline ea & 500 & 960 & 500 & 070 & 07.5 & 830 & 330 & 010 & 340 & 27.5 & 830 & 870 & 510 & 170 & 95 \\
\hline $\mathrm{n}$ & & & & & & & & & & & & & & & \\
\hline \multicolumn{16}{|c|}{ Net Income (PhP ha $\left.{ }^{-1}\right)$} \\
\hline \multirow[t]{3}{*}{1} & 205 & 264 & 295 & 188 & 2382 & 242 & 248 & 276 & 252 & 2550 & 196 & 181 & 981 & 145 & 1555 \\
\hline & 740 & 040 & 210 & 061. & 62.9 & 290 & 140 & 810 & 961. & 50.4 & 990 & 140 & 60 & 961. & 62.9 \\
\hline & & & & 8 & 5 & & & & 8 & 5 & & & & 8 & 5 \\
\hline \multirow[t]{3}{*}{2} & 244 & 253 & 238 & 228 & 2410 & 268 & 223 & 275 & 235 & 2508 & 208 & 160 & 136 & 137 & 1608 \\
\hline & 376 & 376 & 046 & 397. & 48.9 & 476 & 476 & 496 & 797. & 11.4 & 976 & 626 & 796 & 147. & 86.4 \\
\hline & & & & 8 & 5 & & & & 8 & 5 & & & & 8 & 5 \\
\hline 3 & 258 & 221 & 270 & 190 & 2351 & 247 & 231 & 261 & 212 & 2381 & 169 & 216 & 144 & 130 & 1653 \\
\hline & 200 & 550 & 220 & 521. & 22.9 & 300 & 450 & 820 & 121. & 72.9 & 450 & 300 & 820 & 971. & 85.4 \\
\hline & & & & 8 & 5 & & & & 8 & 5 & & & & 8 & 5 \\
\hline 4 & 189 & 233 & 254 & 232 & 2274 & 207 & 245 & 239 & 231 & 2310 & 175 & 214 & 167 & 123 & 1703 \\
\hline & 650 & 000 & 170 & 821. & 10.4 & 900 & 400 & 920 & 071. & 72.9 & 900 & 250 & 920 & 221. & 22.9 \\
\hline & & & & 8 & 5 & & & & 8 & 5 & & & & 8 & 5 \\
\hline 5 & 271 & 224 & 232 & 138 & 2165 & 242 & 222 & 311 & 300 & 2694 & 170 & 219 & 178 & 102 & 1679 \\
\hline
\end{tabular}




\begin{tabular}{|l|c|c|c|c|c|c|c|c|c|c|c|c|c|c|c|}
\hline & 076 & 376 & 246 & 697. & 98.9 & 726 & 726 & 396 & 897. & 36.4 & 626 & 826 & 496 & 947. & 73.9 \\
8 & 5 & & & & 8 & 5 & & & & 8 & 5 \\
\hline M & 233 & 239 & $\mathbf{2 5 7}$ & 195 & 2316 & 241 & 234 & $\mathbf{2 7 3}$ & 246 & 2489 & 184 & $\mathbf{1 9 8}$ & 145 & 128 & 1640 \\
ea & 808. & 268. & $\mathbf{9 7 8}$ & 700. & 88.8 & 738. & 238. & $\mathbf{0 8 8}$. & 570. & 08.8 & 388. & $\mathbf{4 2 8 .}$ & 238. & 050. & 26.3 \\
$\mathrm{n}$ & 4 & 4 & $\mathbf{4}$ & 2 & 5 & 4 & 4 & $\mathbf{4}$ & 2 & 5 & 4 & $\mathbf{4}$ & 4 & 2 & 5 \\
\hline \multicolumn{10}{|c|}{ Return on Investment (PhP return per peso invested) } \\
\hline 1 & 4.32 & 5.02 & 5.23 & 3.12 & 4.42 & 5.17 & 4.78 & 4.97 & 4.25 & 4.79 & 3.21 & 2.73 & 1.40 & 1.97 & 2.33 \\
\hline 2 & 5.17 & 4.85 & 4.24 & 3.81 & 4.52 & 5.77 & 4.34 & 4.98 & 3.98 & 4.77 & 3.42 & 2.43 & 1.96 & 1.86 & 2.42 \\
\hline 3 & 5.33 & 4.14 & 4.72 & 3.12 & 4.33 & 5.18 & 4.39 & 4.63 & 3.51 & 4.43 & 2.72 & 3.22 & 2.04 & 1.75 & 2.43 \\
\hline 4 & 3.96 & 4.41 & 4.48 & 3.85 & 4.18 & 4.41 & 4.71 & 4.29 & 3.87 & 4.32 & 2.86 & 3.22 & 2.38 & 1.66 & 2.53 \\
\hline 5 & 5.73 & 4.29 & 4.14 & 2.31 & 4.12 & 5.13 & 4.26 & 5.55 & 5.02 & 4.99 & 2.80 & 3.33 & 2.56 & 1.40 & 2.52 \\
\hline M & 4.9 & 4.54 & 4.56 & 3.24 & & 5.13 & 4.5 & 4.88 & 4.13 & & 3.00 & 2.99 & 2.07 & 1.73 & \\
ea & & & & & & & & & & & & & & \\
n & & & & & & & & & & & & & \\
\hline
\end{tabular}

Formula : Total cost of production=total cash and non-cash; Gross income $=$ YieldxPrice $/ \mathrm{kg}$

Net income $=$ Gross income-Total production cost $; \quad$ ROI $=$ Net income $/$ Total Production

Cost 


\section{Cost and Return Analysis}

The parameters used were total production cost, gross income, net income and return on investment (ROI) as shown in Table 9. The analysis was done to determine the economic viability of each genotype undervarying fertilizer treatments across all sites. Cash and non-cash cost were recorded and considered as total cost. Pre-land preparation, material and labor costs were included. Gross income was computed by multiplying the total produce in each treatment per genotype with the price per kilogram yield $=$ PhP60per kg. The net income was obtained by subtracting the total production cost from the gross income. The return on investment was also determined per treatment per genotype by dividing the net income by the total cost multiplied by 100 . Thus, ROI reflects the amount of return per peso invested for each treatment (Table 9).

Site 1(Sarrat, Ilocos Norte).The highest total production costin this site was obtained from theplots applied with inorganic fertilizer $(\mathrm{PhP}$ 60,370), while the lowest was the control ( $\mathrm{PhP}$ 47,692). Among the genotypes used, Genotype 3 incurred the highest $(\mathrm{PhP} 55,077)$ total production cost, while Genotype 2 and Genotype 5 were equally the lowest. Genotype 3 is large-seeded, thus less number of seeds are contained per $\mathrm{kg}$, such that heavier weight of planting material is needed to satisfy the seed requirement (e. g. 21,000 seeds per $\mathrm{ha}^{-1}$ ), more were used in terms of kilogram, thus, higher seed cost. Highest gross income was obtained in plants with 50\% IF + 50\% OF ( $\mathrm{PhP} 314,500$, while the lowest was in IF applied treatment ( $\mathrm{PhP} 256,070)$. Genotype 2 had the highest gross income ( $\mathrm{PhP} 294,950$ ), while Genotype 5 had ( $\mathrm{PhP} 270,500)$, the lowest in yield.

Plants applied with 50\% OF $+50 \%$ IF produced the highest net income (PhP 257879), while the lowest was obtained in IF applied plants (PhP 195,700). Among genotypes, the highest net income was obtained in Genotype 2 ( $\mathrm{PhP} 241049$ ), while the lowest was in Genotype 5 (PhP 216599).

Control plants had the highest ROI (4.9), while the IF applied plants had the lowest ROI (3.24). Among the genotypes, the highest ROI was from Genotype 2 and the lowest was from Genotype 5.
Site 2 (Dingras, Ilocos Norte. Among the fertilizer treatments, the highest total production cost in this site was obtained in treatment with inorganic fertilizer application (PhP 59770), while the lowest was from the unfertilized treatment with $\mathrm{PhP}$ 47,092. Among the genotypes, the highest was incurred by Genotype 3 (PhP 54,327), while the lowest was from Genotype 2 (PhP 53,151).

The highest was obtained from plants applied with $50 \%$ OF $+50 \%$ IF (P329010), while the lowest was with OF application ( $\mathrm{PhP}$ 286,330). For the genotypes, the highest gross was obtained from Genotype 5 (PhP 323,337) and the lowest was from Genotype 4 with $\mathrm{PhP}$ 284800. In this case Genotype 5 had the highest root yield so it had the highest income.

The highest net income per hectare, was obtained in plants applied with 50\% OF + 50\% IF (PhP 273,088) and the lowest was with OF (PhP 234,238). With regards to genotypes, Genotype5 had the highest net income (PhP 269,436) and the lowest was Genotype 4 with PhP 231,073. High net income in Genotype 5 is due to its high yield while low net income of Genotype 4 was due to its low yield.

The ROI was highest in unfertilized plants (5.13)and the lowest was with IF (4.13). Among the genotypes, the highest was from Genotype 5 (4.99) and the lowest was from Genotype 4 with 4.32 .

Site 3 (Bangui, Ilocos Norte).Similar to the other sites, the highest total cost of production in this site was obtained from plants applied with inorganic fertilizer (PhP 74,120) and the lowest was with the unfertilized plants ( $\mathrm{PhP}$ 61,442). Among the genotypes, the highest was incurred by Genotype 3 (PhP 68,827) while the lowest was from Genotype 2 and Genotype 5.

For the gross income, the highest was computed from the plants applied with organic fertilizer (PhP264870) due to higher yield while the lowest was with inorganic fertilizer (PhP202170). Among the genotypes, Genotype 4 got the highest gross with PhP 238,550 while G1 had the lowest with $\mathrm{PhP} 223,550$. 
Plants applied with organic fertilizer had the highest net income ( $\mathrm{PhP}$ 198,428), while the lowest was from the plants applied with inorganic fertilizer with PhP128050 due to the high production cost incurred in their fertilizer treatment.

The highest ROI was obtained from the unfertilized plants (3.00) but is comparable with the plants applied with organic fertilizer (2.99), and the lowest was with inorganic fertilizer (1.73). For the genotypes, the highest ROI was from obtained from Genotype 4 (2.53) and the lowest was from Genotype 1 with 2.33 .

Considering the fertilizer treatments, the application of inorganic fertilizer incurred the highest production cost and lowest returns. This is attributed to the high cost of synthetic chemical fertilizer materials used as compared to other locally available fertilizer materials such as organic fertilizer and its cost is minimal. The use of organic fertilizer material usually improve the soil characteristics as well as microbial $\mathrm{N}$ fixation of the plant. Application of organic fertilizer alone or in combination with inorganic fertilizer was found to increased yield at lesser cost particularly in Site 3.

On the other hand, unfertilized plants appeared to have comparable with the high results obtained from organic fertilization. This proves that even without added fertilizer, yam bean plants can still produce with returns because this plant being a legume, has the ability to fix for its own food nutrient using the available material from the soil and its environment. However, for purposes of soil improvement especially in the areas which are less productive like in Site 3, the use of OF is favored.

\section{Energy Utilization and Accounting}

The parameters used include the data on inputs both direct (e.g. fuel \& oil, machineries, fertilizers, pesticides) and indirect (e.g. seeds and labor)as well as the farm operations that include activities from pre-land preparation, fertilization, planting, care and management of the crop, harvesting and post harvest activities considered in man-days as well as man-animal days. This determines the energy cost in producing the yield of your bean genotypes grown under the different fertilizer treatments in each site in terms of energy used in addition to the cost of implementation as well as inputs used. Energy utilization was computed on a hectare-basis and liter diesel oil equivalent per $\mathrm{kg}$ of produced (yield).

Site 1(Sarrat, Ilocos Norte).Table 10 shows the summary of the result of energy accounting for growing yam bean genotypesunder different fertilizer treatments. Among the fertilizer treatments used in this site, the application of inorganic fertilizer had the highest energy spent per kilogram yam bean produced, with $8.53 \mathrm{~L}$ diesel oil equivalent ( $\mathrm{LDOE} \mathrm{kg}^{-1}$ ), this was followed by plants applied with $50 \% \mathrm{OF}+50 \% \mathrm{IF}$ fertilizer treatment (4.43 LDOE $\mathrm{kg}^{-1}$ ) and the lowest was with the plants applied with organic fertilizer (2.18 LDOE $\mathrm{kg}^{-1}$ ).

The high energy spent by using inorganic fertilizer can be attributed to the use of synthetic fertilizers wherein, for a given volume of produce, an expenditure of oil during the manufacture of the production inputs, and the embedded energy cost of machineries and its fuel requirement to perform a specific function were included. To manufacturer $1 \mathrm{~kg}$ of $\mathrm{N}$ fertilizer for example, would require 1.4 -1.8 to 2.4 LDOE, this excludes the transport cost from its area of origin to its destination (Pimentel, 1980). Hence, the high cost of this fertilizer material.

Site 2 (Dingras, Ilocos Norte). Similar to Site 1, the highest energy spent in this site was obtained in plants applied with IF (7.05 LDOE $\left.\mathrm{kg}^{-1}\right)$, while $50 \% \mathrm{OF}+50 \% \mathrm{IF}$ follows with $4.24 \mathrm{LDOE} \mathrm{kg}^{-1}$. The high cost of this fertilizer treatment is attributed to the presence of $\mathrm{N}$ fertilizer material which is $50 \%$. The lowest was with unfertilized plants (2.19 $\mathrm{LDOE} \mathrm{kg}^{-1}$ ).

Site 3 (Bangui, Ilocos Norte).Generally, this site had the highest energy spent among the three sites used. The high cost in this site is attributed to the additional cost incurred considering the distance of the experimental site to the place where inputs are taken including transport cost and labor in hauling. In addition, the lower overall yield than the other two sites is also a factor of its high energy expenditure. In addition, the quality of the soil based on pre-plant analysis which is lower in some important soil factors as OM, as well as the unusual 
climatic condition contributed to lower yield, thus affecting the energy cost of the crop. Among the fertilizer treatments, plants applied with IF had the highest energy expenditure (10.65 LDOE kg-1), while the lowest is the OF applied plants $(2.42$ LDOE $\mathrm{kg}^{-1}$ ). The low cost incurred by the plants applied with organic fertilizer alone is due to the availability of this fertilizer material at the different sites. The cost was negligible or even free in Site 3. Also, the soil improvement resulted from application of this fertilizer material as well as its contribution to increasing microbial population to the plant itself for microbial $\mathrm{N}$ fixation resulted to increased yield at low energy cost.

Among the genotypes used, Genotype 3spent the highest energy in all the sites while the lowest was Genotype 5.Genotype 3is a large-seeded genotype, therefore higher weight of planting matter is needed to satisfy the seeding requirement which is based on number of seeds per unit area. On the other hand, Genotype 5 has smaller seeds, so lower weight of planting material was used to satisfy the requirement of the area, resulting to lower energy expenditure.

Table 11 shows the specific site and genotype as affected by fertilizer treatments. Generally, based from the results, fertilizer treatments significantly affected the energy utilization of yam bean plants in this experiment. Plants applied with inorganic fertilizer treatment had the highest energy expenditure (8.62 $\left.\mathrm{LDOE} \mathrm{kg}^{-1}\right)$. Plants applied with organic fertilizer had the lowest energy spent in yam bean production in terms of LDOE per $\mathrm{kg}$ of yam bean produced (1.97 LDOE $\mathrm{kg}^{-1}$ ) The low cost incurred by the plants applied with organic fertilizer alone is accounted by the availability of this fertilizer material in the different sites. The cost was negligible or even free especially in Site 3.

The application of organic fertilizer had contributed to the improvement of the soil through soil structure improvement as well as the plant itself. Being a legume, yam been has the capacity to fix microbial $\mathrm{N}$ for its needs. With the addition of organic fertilizer. microbial population for $\mathrm{N}$ fixation in the plant is increased. Among the genotypes used in this site, Genotype 1 was found out to have high yields but lowest in energy expenditure. Genetically, Genotype 1 is smallseeded, thus lesser number of seeds (in terms of weight) and thus lower energy equivalent was used.

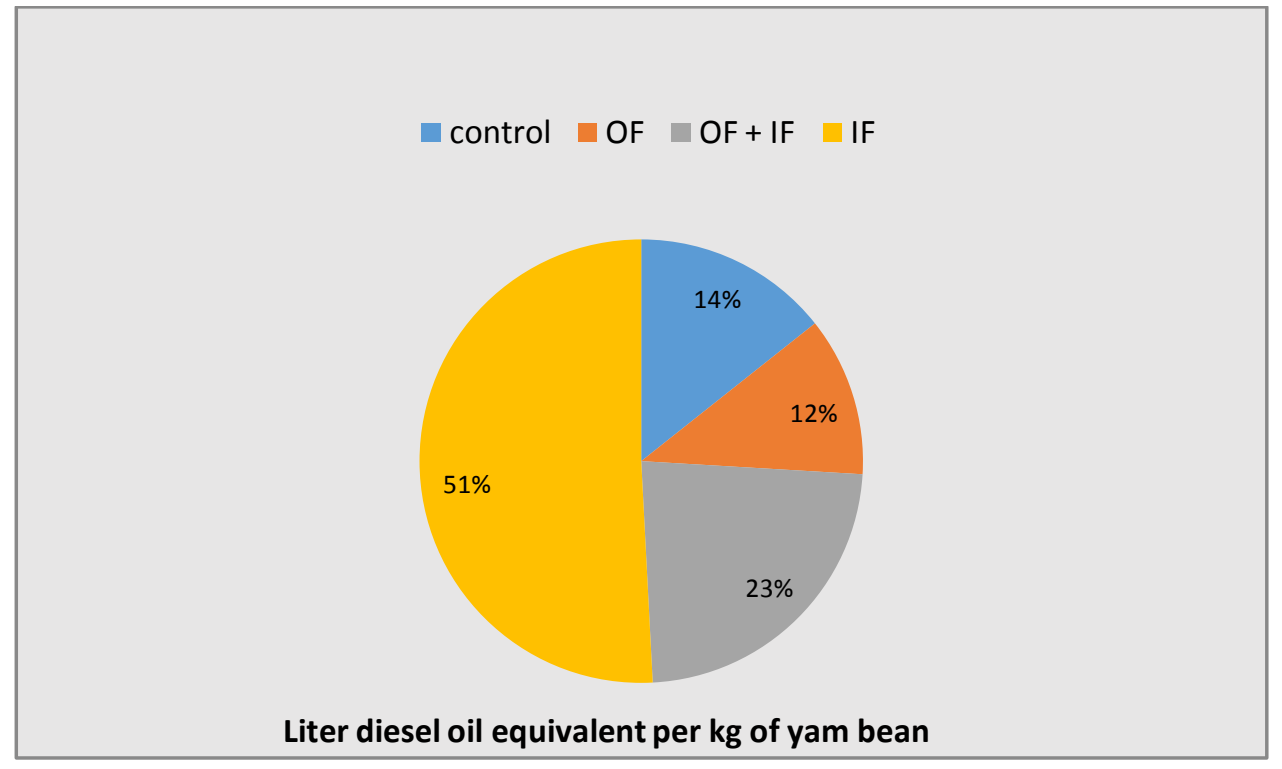

This figure shows the energy expenditure of yam bean Genotype 1 grown in Site 1 (Sarrat, Ilocos Norte). The application of organic fertilizer spent only $12 \%$ of the total energy expenditure, followed by the plants applied with $50 \% \mathrm{OF}+50 \% \mathrm{IF}$, while the highest was those applied with inorganic fertilizer, with $51 \%$ of the total energy expenditure. 
With respect to energy productivity in this site, the use of organic fertilizer turned out to be the most productive in terms of yield over the energy used with $0.51 \mathrm{~kg}$ per LDOE, while the least productive was observed from plants with inorganic fertilizer (1 liter of oil is PhP 52). On energy intensity, the use of organic fertilizer had the lowest energy intensity (1.97 LDOE per kg).

Energy intensity on the other hand refers to the amount of energy used per unit of activity. In here, the use of organic fertilizer showed the lowest energy intensity $(1.97 \mathrm{LDOE} / \mathrm{kg})$, while the highest was with inorganic fertilizer application with $8.62 \mathrm{LDOE} / \mathrm{kg}$ ). These mean that applying organic fertilizer results to high quality produced at lower quantity of energy spent and lower energy spent for a higher production. 
Table 10. Summary table on energy accounting of yam bean grown with four fertilizer treatments in three sites of Ilocos Norte Philippines. 2010-2011 Cropping Season

\begin{tabular}{|c|c|c|c|c|c|c|c|c|c|c|c|c|c|c|c|}
\hline \multicolumn{16}{|c|}{ Total Production Liter Diesel Oil Equivalent $\left(\mathrm{kg}^{-1}\right)$} \\
\hline $\mathbf{G}$ & \multicolumn{5}{|c|}{ SITE 1} & \multicolumn{5}{|c|}{ SITE 2} & \multicolumn{5}{|c|}{ SITE 3} \\
\hline & $\begin{array}{l}\text { CO } \\
\text { NT } \\
\text { RO } \\
\text { L }\end{array}$ & $\begin{array}{l}\text { OR } \\
\text { GA } \\
\text { NIC }\end{array}$ & $\begin{array}{l}\mathbf{5 0} \\
\% \\
\text { OF } \\
+5 \\
0 \\
\% \text { I } \\
\text { F }\end{array}$ & $\begin{array}{l}\text { INO } \\
\text { RGA } \\
\text { NIC }\end{array}$ & $\begin{array}{l}\mathbf{M} \\
\mathbf{E} \\
\mathbf{A} \\
\mathbf{N}\end{array}$ & $\begin{array}{l}\text { CO } \\
\text { NT } \\
\text { RO } \\
\text { L }\end{array}$ & $\begin{array}{l}\text { OR } \\
\text { GA } \\
\text { NIC }\end{array}$ & $\begin{array}{l}50 \\
\% \\
\text { OF } \\
+5 \\
0 \\
\% \text { I } \\
\text { F }\end{array}$ & $\begin{array}{l}\text { INO } \\
\text { RGA } \\
\text { NIC }\end{array}$ & $\begin{array}{l}\mathbf{M} \\
\mathbf{E} \\
\mathbf{A} \\
\mathbf{N}\end{array}$ & $\begin{array}{l}\text { CO } \\
\text { NT } \\
\text { RO } \\
\text { L }\end{array}$ & $\begin{array}{l}\text { OR } \\
\text { GA } \\
\text { NIC }\end{array}$ & $\begin{array}{l}\mathbf{5 0} \\
\% \\
\text { OF } \\
+5 \\
0 \\
\% \text { I } \\
\text { F }\end{array}$ & $\begin{array}{l}\text { INO } \\
\text { RGA } \\
\text { NIC }\end{array}$ & $\begin{array}{l}\mathbf{M} \\
\mathbf{E} \\
\mathbf{A} \\
\mathbf{N}\end{array}$ \\
\hline 1 & 2.45 & 1.97 & $\begin{array}{c}3.9 \\
4\end{array}$ & 8.62 & $\begin{array}{l}4.2 \\
4\end{array}$ & 2.18 & 2.12 & $\begin{array}{c}4.1 \\
7\end{array}$ & 6.85 & $\begin{array}{l}3.8 \\
3\end{array}$ & 2.43 & 2.56 & $\begin{array}{c}8.2 \\
4\end{array}$ & 9.73 & $\begin{array}{l}5.7 \\
4\end{array}$ \\
\hline 2 & 2.14 & 2.06 & $\begin{array}{c}4.7 \\
0\end{array}$ & 7.40 & $\begin{array}{l}4.0 \\
8\end{array}$ & 1.98 & 2.29 & $\begin{array}{c}4.1 \\
8\end{array}$ & 7.24 & $\begin{array}{l}3.9 \\
2\end{array}$ & 2.31 & 2.78 & $\begin{array}{c}6.6 \\
9\end{array}$ & 10.13 & $\begin{array}{l}5.4 \\
8\end{array}$ \\
\hline 3 & 2.09 & 2.35 & $\begin{array}{c}4.2 \\
7\end{array}$ & 8.55 & $\begin{array}{l}4.3 \\
2\end{array}$ & 2.17 & 2.28 & $\begin{array}{c}4.4 \\
0\end{array}$ & 7.90 & $\begin{array}{l}4.1 \\
9\end{array}$ & 2.77 & 2.28 & $\begin{array}{c}6.4 \\
8\end{array}$ & 10.45 & $\begin{array}{l}5.4 \\
9\end{array}$ \\
\hline 4 & 2.66 & 2.23 & $\begin{array}{c}4.4 \\
7\end{array}$ & 7.31 & $\begin{array}{l}4.1 \\
7\end{array}$ & 2.48 & 2.15 & $\begin{array}{c}4.7 \\
0\end{array}$ & 7.37 & $\begin{array}{l}4.1 \\
8\end{array}$ & 2.66 & 2.27 & $\begin{array}{c}5.8 \\
3\end{array}$ & 10.85 & $\begin{array}{l}5.4 \\
0\end{array}$ \\
\hline 5 & 1.96 & 2.28 & $\begin{array}{c}4.7 \\
9\end{array}$ & 10.75 & $\begin{array}{l}4.9 \\
4\end{array}$ & 2.15 & 2.29 & $\begin{array}{c}3.7 \\
6\end{array}$ & 5.92 & $\begin{array}{l}3.5 \\
3\end{array}$ & 2.69 & 2.20 & $\begin{array}{c}5.5 \\
7\end{array}$ & 12.09 & $\begin{array}{l}5.6 \\
4\end{array}$ \\
\hline $\begin{array}{l}\text { M } \\
\text { E } \\
A \\
N\end{array}$ & 2.46 & 2.18 & $\begin{array}{l}4.4 \\
3\end{array}$ & 8.53 & & 2.19 & 2.23 & $\begin{array}{l}4.2 \\
4\end{array}$ & 7.05 & & 2.57 & 2.42 & $\begin{array}{l}6.5 \\
6\end{array}$ & 10.65 & \\
\hline
\end{tabular}


Table 11. Energy (LDOE) accounting of yam bean with four fertilizer treatments in Sarrat, Ilocos Norte. 2008-2009 Cropping Season

\begin{tabular}{|c|c|c|c|c|c|c|c|c|}
\hline \multirow[b]{3}{*}{ ITEM } & \multicolumn{8}{|c|}{ Genotype 1} \\
\hline & \multicolumn{2}{|c|}{ Control } & \multicolumn{2}{|c|}{ Organic $(100 \%)$} & \multicolumn{2}{|c|}{$50 \% \mathrm{OF}: 50 \% \mathrm{IF}$} & \multicolumn{2}{|c|}{ Inorganic $(100 \%)$} \\
\hline & LDOE & Percent & LDOE & Percent & LDOE & Percent & LDOE & Percent \\
\hline \multicolumn{9}{|l|}{ A. Seed Production } \\
\hline Land preparation $\left(4.5^{*} 18\right) / 11.414$ & 7.10 & 5.75 & 7.10 & 5.70 & 7.10 & 2.56 & 7.10 & 1.66 \\
\hline Planting of roots, $5 \mathrm{MD}(5 * 8 * .549) / 11.414$ & 1.92 & 1.56 & 1.92 & 1.55 & 1.92 & 0.69 & 1.92 & 0.45 \\
\hline Roots, $(30.4 * 1.14) / 11.414$ & 3.60 & 2.91 & 3.60 & 2.89 & 3.60 & 1.30 & 3.60 & 0.84 \\
\hline $\begin{array}{l}\text { Trellising \& Cultivation,2 MD } \\
(2 * 8 * .549) / 11.414\end{array}$ & 0.77 & 0.62 & 0.77 & 0.62 & 0.77 & 0.28 & 0.77 & 0.18 \\
\hline $\begin{array}{l}\text { Harvesting and threshing (seeds) } 10 \\
\text { MD*8*.549 }\end{array}$ & 3.85 & 3.12 & 3.85 & 3.09 & 3.85 & 1.39 & 3.85 & 0.90 \\
\hline \multicolumn{9}{|l|}{ B. Plant Crop } \\
\hline $\begin{array}{l}\text { Land preparation (Machinery) } \\
(4.5 * 18) / 11.414\end{array}$ & 7.10 & 5.75 & 7.10 & 5.70 & 7.10 & 2.56 & 7.10 & 1.66 \\
\hline Fuel & 51.00 & 41.34 & 51.00 & 40.96 & 51.00 & 18.38 & 51.00 & 11.92 \\
\hline $\begin{array}{l}\text { Planting, } 15 \mathrm{MD} \text { (to include basal fertilizer } \\
\text { appln.) }\end{array}$ & 5.77 & 4.68 & 5.77 & 4.64 & 5.77 & 2.08 & 5.77 & 1.35 \\
\hline Seeds, $(18 \mathrm{~kg} * 1.7) / 11.414$ & 0.25 & 0.21 & 0.25 & 0.20 & 2.68 & 0.97 & 2.68 & 0.63 \\
\hline $\begin{array}{l}\text { Field Lay-out (to include making of plots), } 10 \\
\text { MD }\end{array}$ & 3.85 & 3.12 & 3.85 & 3.09 & 3.85 & 1.39 & 3.85 & 0.90 \\
\hline Fertilizer (fertilizer + hauling), $3 \mathrm{MD}$ & & & 1.15 & 0.93 & 1.15 & 0.42 & 1.15 & 0.27 \\
\hline Nitrogen $(214.28 * 14.3) / 11.414$ & & & & & 134.23 & 48.38 & 268.46 & 62.73 \\
\hline Phosphorous $(150 * 1.6) / 11.414$ & & & & & 15.11 & 5.45 & 30.23 & 7.06 \\
\hline Potassium $(16.66 * 1.6) / 11.414$ & & & & & 1.17 & 0.42 & 2.34 & 0.55 \\
\hline Pesticides, Tamaron, 2 lit $(2 * 7.61) / 11.414$ & 1.33 & 1.08 & 1.33 & 1.07 & 1.33 & 0.48 & 1.33 & 0.31 \\
\hline Labor, $2 \mathrm{MD}(2 * 8 * .549) / 11.414$ & 0.77 & 0.62 & 0.77 & 0.62 & 0.77 & 0.28 & 0.77 & 0.18 \\
\hline Cultivation/handweeding, 20 MD*.549 & 7.70 & 6.24 & 7.70 & 6.18 & 7.70 & 2.77 & 7.70 & 1.80 \\
\hline Irrigation, NIA, 227mcal/11.414 & 19.89 & 16.12 & 19.89 & 15.97 & 19.89 & 7.17 & 19.89 & 4.65 \\
\hline
\end{tabular}


Jocelyn A. Bernabe / Energy utilization of yam bean....

\begin{tabular}{|l|r|r|r|r|r|r|r|r|} 
Labor, 2 MD*8*.549 & 0.77 & 0.62 & 0.77 & 0.62 & 0.77 & 0.28 & 0.77 & 0.18 \\
\hline Harvesting (roots) 20 MD*8*.549 & 7.70 & 6.24 & 7.70 & 6.18 & 7.70 & 2.77 & 7.70 & 1.80 \\
\hline Total Root Production LDOE/ha & 123.36 & 100.00 & 124.51 & 100.00 & 277.45 & 100.00 & 427.96 & 100.00 \\
\hline Total Root Production LDOE/kg & 2.43 & & 1.97 & & 3.94 & & 8.62 & \\
\hline yield/ha & 50.67 & & 63.33 & & 70.33 & & 49.67 & \\
\hline & & & & & & & & \\
\hline $\begin{array}{l}\text { Energy productivity (yield output/energy } \\
\text { input) }\end{array}$ & 0.41 & & 0.51 & & 0.25 & & 0.12 & \\
\hline Energy Intensity (energy input/yield output) & 2.43 & & 1.97 & & 3.94 & & 8.62 & \\
\hline
\end{tabular}

Legend: $\mathrm{LDOE}=$ liter diesel oil equivalent

$$
1 \mathrm{LDOE}=11.414 \mathrm{Mcal}
$$

Note: The number of unit indicated in the table is multiplied by the respective energy equivalent as shown below divide by 11.414 to get LDOE

Nitrogen $=14.3 \mathrm{LDOE}$

Phosphrous $=2.3 \mathrm{LDOE}$

Potassium $=1.6 \mathrm{LDOE}$

Labor $=0.549 \mathrm{Mcal}$

Seeds $=1.7 \mathrm{Mcal}$

Roots $=1.14 \mathrm{Mcal}$

Organic Fertilizer applied: Decomposed chicken manure

Nitrogen $=3.23$

Phosphorous $=4.27$

Potassium $=2.54$

Table 12. Energy $(L D O E)$ cost $(P h P)^{*}$ of yam bean with four fertilizer treatments in Ilocos Norte.

2010-2011 Cropping Season.

\begin{tabular}{|c|c|c|c|c|c|c|c|c|}
\hline & \multicolumn{9}{|c|}{ Genotype 1 } \\
\hline & \multicolumn{2}{|c|}{ Control } & \multicolumn{2}{|c|}{ Organic (100\%) } & \multicolumn{2}{|c|}{$50 \%$ OF: 50\% IF } & \multicolumn{2}{|c|}{ Inorganic (100\%) } \\
\hline ITEM & LDOE & $\begin{array}{c}\text { Cost } \\
(\mathrm{P})\end{array}$ & LDOE & $\begin{array}{c}\text { Cost } \\
(\mathrm{P})\end{array}$ & LDOE & Cost (P) & LDOE & Cost (P) \\
\hline A. Seed Production & & & & & & & & \\
\hline
\end{tabular}




\begin{tabular}{|c|c|c|c|c|c|c|c|c|}
\hline Land preparation & 7.1 & 369.2 & 7.1 & 369.2 & 7.1 & 369.2 & 7.1 & 369.2 \\
\hline Planting of roots & 1.92 & 99.84 & 1.92 & 99.84 & 1.92 & 99.84 & 1.92 & 99.84 \\
\hline Roots & 3.6 & 187.2 & 3.6 & 187.2 & 3.6 & 187.2 & 3.6 & 187.2 \\
\hline Trellising \& Cultivation, $2 \mathrm{MD}$ & 0.77 & 40.04 & 0.77 & 40.04 & 0.77 & 40.04 & 0.77 & 40.04 \\
\hline Harvesting and threshing (seeds) & 3.85 & 200.2 & 3.85 & 200.2 & 3.85 & 200.2 & 3.85 & 200.2 \\
\hline \multicolumn{9}{|l|}{ B. Plant Crop } \\
\hline Land preparation (Machinery) & 7.1 & 369.2 & 7.1 & 369.2 & 7.1 & 369.2 & 7.1 & 369.2 \\
\hline Fuel & 51 & 2652 & 51 & 2652 & 51 & 2652 & 51 & 2652 \\
\hline Planting & 5.77 & 300.04 & 5.77 & 300.04 & 5.77 & 300.04 & 5.77 & 300.04 \\
\hline Seeds & 0.25 & 13 & 0.25 & 13 & 2.68 & 139.36 & 2.68 & 139.36 \\
\hline Field Lay-out (to include making of plots) & 3.85 & 200.2 & 3.85 & 200.2 & 3.85 & 200.2 & 3.85 & 200.2 \\
\hline Fertilizer (fertilizer + hauling) & & & 1.15 & 59.8 & 1.15 & 59.8 & 1.15 & 59.8 \\
\hline Nitrogen & & & & & $\begin{array}{r}134.2 \\
3 \\
\end{array}$ & 6979.96 & $\begin{array}{r}268.4 \\
6 \\
\end{array}$ & 13959.92 \\
\hline Phosphorous & & & & & 15.11 & 785.72 & 30.23 & 1571.96 \\
\hline Potassium & & & & & 1.17 & 60.84 & 2.34 & 121.68 \\
\hline Pesticides, Tamaron & 1.33 & 69.16 & 1.33 & 69.16 & 1.33 & 69.16 & 1.33 & 69.16 \\
\hline Labor & 0.77 & 40.04 & 0.77 & 40.04 & 0.77 & 40.04 & 0.77 & 40.04 \\
\hline Cultivation/handweeding & 7.7 & 400.4 & 7.7 & 400.4 & 7.7 & 400.4 & 7.7 & 400.4 \\
\hline Irrigation, NIA & 19.89 & 1034.28 & 19.89 & $\begin{array}{r}1034.2 \\
8\end{array}$ & 19.89 & 1034.28 & 19.89 & 1034.28 \\
\hline Labor & 0.77 & 40.04 & 0.77 & 40.04 & 0.77 & 40.04 & 0.77 & 40.04 \\
\hline Harvesting (roots) & 7.7 & 400.4 & 7.7 & 400.4 & 7.7 & 400.4 & 7.7 & 400.4 \\
\hline Total Root Production LDOE/ha & 123.36 & 6414.72 & 124.51 & $\begin{array}{r}6474.5 \\
2\end{array}$ & $\begin{array}{r}277.4 \\
5\end{array}$ & 14427.4 & $\begin{array}{r}427.9 \\
6\end{array}$ & 22253.92 \\
\hline Total Root Production LDOE/kg & 2.43 & 126.36 & 1.97 & 102.44 & 3.94 & 204.88 & 8.62 & 448.24 \\
\hline
\end{tabular}

*Price per liter in Ilocos Norte is PhP52.0 
The energy utilization is highest using inorganic fertilizer. Among the fertilizer materials used, the use of nitrogen fertilizer which incurred about $62.73 \%$ (which is more than half of the total energy spent), accounted the highest amount of energy bill which means that it is the main fossil fuel energy utilizing agrochemical input in addition to the cost in its manufacture which requires $2.4 \mathrm{LDOE}$ aside from the transport cost incurred (Pimentel 1980). This is then the reason why farmers do not have too much or even negligible net when farming especially with the use of inorganic chemical inputs

Therefore in order to reduce the energy cost incurred in yam bean production, it is necessary to look into some alternatives like for example the use of organic fertilizer such as decomposed chicken manure. This does not only reduce energy, monetary cost but also improves the soil properties in terms of its water and nutrient holding capacity as well as very useful to the plant especially that yam bean is a legume crop and has the capability to fix microbial $\mathrm{N}$ with the presence of added microbes from the organic fertilizer. As seen in Table 19, the energy cost of producing a kilo of yam bean using organic fertilizer is only 1.97 LDOE, with energy productivity of 0.51 . In Table 20 , it shows that the cost of energy spent using organic fertilizer was only PhP102.44 per kilo of yam bean produced.

The application of $50 \% \mathrm{OF}+50 \% \mathrm{IF}$ produced the highest net income in Site $1(\mathrm{PhP} 257,978)$ and 2 (PhP273,088). This is attributed to reduction of cost of fertilizer and high yield under this treatment. Highest net income was obtained in Genotype 2 for Site 1, while Genotype 5 for Sites 2 and 3 , which is attributed to the high yields of this variety in these sites. The ROI across sites is constantly highest in unfertilized plants, which reflects low cost of production under low yield levels, while highest ROI was obtained in IF application, although this is within the high yield levels. The application of IF is the most energy consuming in producing yam bean, as reflected in the highest LDOE. The lowest LDOE was obtained in the unfertilized yam bean crop.

\section{SUMMARY AND CONCLUSION}

The three selected sites represented the three dominant soil series (Umingan clay loam, San Manuel silt loam and San Fernando clay) in the yam bean growing areas in Ilocos Norte. The four fertilizer treatments used were: control (no fertilizer applied), organic fertilizer (OFdecomposed chicken manure), $50 \%$ organic fertilizer $(\mathrm{OF})+50 \%$ inorganic fertilizer (IF), and inorganic fertilizer (IF) using the recommended rate in the province which is $35-60-40 \mathrm{~kg} \mathrm{NPK} \mathrm{ha}^{-1}$. All the fertilizer materials were applied before planting. The five yam bean genotypes designated as Genotype 1, Genotype 2, Genotype 3, Genotype 4 , and Genotype 5 were selected based on their availability and initial performance in the area. Initially, these were characterized using available descriptor's list for yam bean.

Six months before the study proper, seed production was done on the five genotypes used. Regular monitoring of the areas was done during the entire duration of the experiment. The data gathered were: pre-plant soil analysis $(\mathrm{pH}$, organic matter and nutrient status of the soils, climatic characteristics before planting and throughout the duration of the study; agronomic parameters which include data on days to germination, flowering and maturity; root characteristics which include root length, diameter, root fresh and dry weights; shoot characteristics consisting of number of branches, shoot weight, and shoot dry weight; growth parameters which include crop growth rate, dry matter production and harvest index; yield; plant nutrient concentration and uptake, nutrient-use efficiency, energy utilization analysis and accounting; and, cost and return analysis.Climatic data were taken from two sources: from the PAGASA, Laoag International Airport and North Wind Power Development Corporation-Bangui Bay Wind Power Project.

Based on the soil analysis the study sites have varying properties. Sites 1 and 2, have almost similar in their soil and climatic characteristics in terms of $\mathrm{P}$ and $\mathrm{K}$, while Site 1 have high $\mathrm{OM}$ and have clayey soil texture unlike Site 2 which has silty soil texture. Site 3 on the other hand is generally sandy, have the lowest OM and appears to be more vulnerable to drought considering the 
erratic rainfall patterns and the slightly higher wind speed during the growing season.

Site 1 (Sarrat, Ilocos Norte). The application of $50 \%$ OF $+50 \%$ IF however, had the best performance in this site. Plants applied with combined OF and IF had the highest yield (62.9 $\mathrm{t}$ $\left.\mathrm{ha}^{-1}\right)$. Thus, the combination of OF and IF produced the highest biomass, had the best biomass partitioning to yield, and had the best conversion of absorbed nutrient ( $\mathrm{N}$ and $\mathrm{P}$ ) to yield formation in this site.

Among genotypes, Genotype 1 performed-well in this site. Although Genotype 1 had the best yield $\left(58.99 \mathrm{t} \mathrm{ha}^{-1}\right)$ in this site, Genotypes 4 and 5 showed good performance, i.e., Genotype 4 (56.38 $\mathrm{t} \mathrm{ha}^{-1}$ ) had the highest HI.

Plants applied with $50 \%$ OF $+50 \%$ IF produced the highest net income (PhP 257,879), while the lowest was in IF applied plants (PhP 195,700). Genotype 2 produced the highest net income ( $\mathrm{PhP} 241,049)$ while the lowest is Genotype 5 ( $\mathrm{PhP} 216,599)$. The lower net income obtained in Genotype 5 was attributed to high cost of the seeds, in spite of its relatively high yield. In terms of ROI, unfertilized plants had an ROI of 4.9, with no cost for fertilizer input, but relatively within the low yield levels. On the other hand, the application of inorganic fertilizer (IF) produced the lowest ROI (3.24). The $50 \%$ OF $+50 \%$ IF had ROI of 4.56 , while producing a high yield level. Among the genotypes, the highest ROI was obtained in Genotype 2, while the lowest was in Genotype 5.

With respect to the energy spent, plants applied with inorganic fertilizer spent the highest with 8.53 LDOE, while the lowest was with OF (2.18 LDOE). Among the genotypes, Genotype 5 incurred the highest with $4.94 \mathrm{LDOE}$, while the lowest was Genotype 2 (4.08 LDOE).

Site 2 (Dingras, Ilocos Norte) Plants applied with $50 \%$ OF $+50 \%$ IF were the earliest to germinate, flower and mature. This fertilizer treatment also produced the heaviest root weight (65.80 t ha-1), all translated to high yield of this treatment in Site 2.

The highest net income was obtained from plants applied with $50 \% \mathrm{OF}+50 \%$ IF (PhP 273,088), while the lowest was from plants applied with $\mathrm{OF}$ (PhP 234, 238). Genotype 5 had the highest net income (PhP 269,436).

The highest ROI was obtained from unfertilized plants (5.13) while the lowest was from plants applied with inorganic fertilizer (1.73). Plants applied with inorganic fertilizer spent the highest energy with 7.05 LDOE while the lowest was in unfertilized plants (2.19 LDOE). Among the genotypes, Genotype 3 had spent the highest energy with 4.19 DOE, while Genotype 5 the lowest with 3.53 LDOE.

Site 3 (Bangui, Ilocos Norte). Organic fertilizer application (OF) was observed to be the best among fertilizer treatments. The application of $50 \%$ $\mathrm{OF}+50 \%$ IF also produced good yield (43.10 t ha1) in this site compared with IF and unfertilized control. While this site is prone to water stress (sand texture, uneven rainfall distribution within the growing season and windy environment), the application of OF alone appears to improve yield as supported by relatively high biomass production, allocation of dry matter to the economic yield, K uptake (as an osmolyte) and high conversion of absorbed $\mathrm{P}$ to yield formation. Genotype 4 had the highest yield in this site (47.71 $\left.\mathrm{t} \mathrm{ha}^{-1}\right)$, which suggests that this genotype may have the tolerance to adverse conditions, like Bangui, Ilocos Norte.

The plants applied with organic fertilizer had the highest net income (PhP 198, 423) while the lowest was from the plants applied with inorganic fertilizer (PhP 128,050). The high net income from the plants applied with inorganic fertilizer is attributed to no cost of fertilizer material while for inorganic, the fertilizer material cost very high. The highest ROI was obtained from unfertilized plants (3.00) and plants applied with organic fertilizer had the lowest ROI of 1.73 .

The highest energy in this site was spent by plants applied with inorganic fertilizer (10.65 LDOE), while the lowest was from plants applied with organic. For the genotypes used, Genotype 1 spent the highest energy (5.74) while the lowest was spent by Genotype 4 with 5.40 LDOE.

Based on the combination of experimental variables, namely fertilizer treatments and genotypes grown in three sites with varying 
edaphic and climatic characteristics, the following generalizations and conclusions were derived from the results:

The application of $50 \% \mathrm{OF}+50 \%$ IF produced the highest yam bean yield in Sites 1 and 2 with relatively good soil characteristics and climatic conditions. The application of OF in Site 3, which is characterized by sandy texture, uneven rainfall, windy condition or in general drought prone areas, produced the highest yield which is supported by the growth and efficiency parameters.

None of the test genotypes had consistent performance (yield) across sites. When the best fertilizer treatment is considered per site, Genotype 1 in Site 1 and genotype 5 in Site 2 performed with application. For Site 3 (drought-prone) Genotype 5 produced the highest yield, as reflected on the growth and nutrient use efficiency parameters. Thus genotype 5 is adapted to favorable as well as unfavorable environments in coupled with appropriate fertilizer application.

The application of $50 \% \mathrm{OF}+50 \%$ IF produced the highest net income in Sites 1 (PhP 257,978) and 2 ( $\mathrm{PhP} 273,088)$. This is attributed to reduction of cost of fertilizer and high yield levels this treatment. Highest net income was obtained in Genotype 2 for Site 1 while Genotype 5 for Sites 2 and 3 , which is attributed to the high yield of this variety in these sites.

The ROI across sites is consistently highest in unfertilized plants, which reflects low cost of production under low yield levels while the highest ROI was obtained in IF application, although this is within the high yield levels.

The application of IF is the most energy consuming in producing yam bean, as reflected in the highest LDOE for this fertilizer practice. The lowest LDOE was obtained in the unfertilized yam bean crop.

Generally, it can be concluded that yam bean production in different growing sites with varying edaphic conditions vary the productivity, cost and return analysis and energy utilization, and as was observed the use of different materials with yam bean genotypes could be grown to particular fertilizer regime.
In Ilocos Norte, with the varying soil types accompanied by unpredictable climatic condition in scattered areas, the use of either organic fertilizer or a combination of organic and inorganic fertilizer can give good returns in the improvement not only the soil condition but also the income of the Ilocanos. Moreso, yam bean crop is a manageable crop and it grows well with these kind of fertilizer materials as was proven by this experiment. In the grass roots of the province, most of the farmers are unable to finance their farming activities, using the available fertilizer material like organic which is just within their vicinity.

\section{REFERENCES}

Ahn, P. M. 1993. Tropical soils and fertilizer use. Longman group, United Kingdom.

Ajibade S. R., B. A.Ogunbodede and B. A.Oyejola. 2002. AMMI analysis of genotypeenvironment interaction for open-pollinated maize varieties evaluated in major agro-ecologies of Nigeria.Tropical agriculture ISSN 0041-3216 CODEN TAGLA2.vol. 79, nº 4, pp. 265-270 [6 page(s) (article)] (14 ref.)

Basilio, S. 2000. Farm machinery estimates for crop production in the Ilocos. Unpublished Paper. MMSU.

Bockari-Gevao, S. M., W. I. Bin Wan Ismail, A. Yahya, and C. C. Wan. 2005. Analysis of energy consumption in lowland rice-based cropping system in Malaysia. J. Sci. Technol., 27(4):819826.

Comstock, R. E. and R. H. Moll. 1963. Genotypeenvironment interaction. In: Statistical Genetics and Plant Breeding (eds. Hanson, W. D. and H. F.Robinson). NAS-NRC Pub. 982 p. 164-196.

Crossa, J. 1990. Statistical analyses of multilocation trials. Adv. Agron. 44:55-85

Duke, J. A. 1981. Legume species: Pachyrhizus erosus, In: Handbook of Legumes of World Economic Importance, pp 182-184. Plenum Press, New York and London.

Escobar, R. 1943. Jicama. Pp 442-444 in Encycloedia Agricola y de Conocimientos Afines...., Vol. 2: Escuela Particular de Agricultura. Cd. Juarez, Chihuahua, Mexico.

Grunenberg, W. J., P. Freynhagen-Leopol \& O. Delgado- Vaquez. 2003. a NEW Yam bean 
(Pachyrhizus spp.) interspecific hybrid. Genetic Resources and Crop Evolution. 50:757-766.

Grunenberg, W. J., F. D. Goffman and L. Velasco. 1999. Characterization of yam bean (Pachyrhizus spp) seeds as a potential source of high palmitic acid oil. Journal of the American Oil Chemists' Society. Springer Berlin/Heidelberg. Vol. 76(11)1309-1312.

Hoof, W.C.H. Van and M. Sorensen. 1989. Pachyrhizus erosus (L.) Urban. Pp.213-215 in Plant Resources of Southeast Asia: A Selection (E. Westphal and P.C.M. Jansen, eds.) Pudoc, Wageningen, The Netherlands.

Huehn, M. 1996. Non-parametric analysis of genotype $\mathrm{x}$ environment interactions by ranks. $\mathrm{p}$. 213-228. In M.S. Kang and H.G. Gauch (ed.) Genotype by environment interaction. CRC Press, Boca Raton, FL.

Meijer, T. M. 1946. The insecticidal constituent of Pachyrhizus erosus Urban, L. - Recueil des travaux chimiques des Pays-Bas. 65:835-842.

Mendoza, T. C. 2007. The Energetics of Ethanol Production and its Implications. Asia Life Sciences 16(2):115-138.

Ozkan, B., H. Agkaoz and C. Fert. 2004. Energy input-output analysis in Turkish agriculture. Renewable Energy 29,39-51.

Pimentel, D. (ed) 1980. Handbook of Energy Utilization in Agriculture. CRC Press, Boca Raton, FL. 474 pp.

Prasad, D. and R. Prakash. 1973. Floral biology of Yam bean, Pachyrhizus erosus(L.) Urban. Indian J. agric. Sci. 43(6):531-535.

Ratanadilok, N. and K. Suriyawan. 1995. Yam bean (Pachyrhizus erosus (L.) Urban) and its economical potential. In: Sorensen M., J. Estrella, O. Hamann \& S. Ruiz, (eds.) (1998): Proceedings of 2nd International Symposium on Tuberous Legumes; Mexico, 5-8 August 1996. macKeenzie, Kobenhavn. Pp. 261-273.

Singh, H., D. Mishra, N. M. Nahar, and M. Ranjan. 2003. Energy use pattern lin production agriculture of a typical village in arid zone India: Part II. Energy Conversion and Management 44(7), 1053-1067.

Sorensen, M. 1990. Observation on distribution, ecology and cultivation of the tuber-bearing legume genus Pachyrhizus Rich, ex DC.
(Fabaceae: Phasoeleae). Wageningen Papers 90$3: 1-38$.

Sorensen, M. 2004. Supercrop: the yam bean, a tuber undaunted by drought, poor soil or insects, produces astonishing yields. The crop is the focus of a worldwide effort to unlock its potential. Natural History.

Sumonsma, J. S. and K. Piluek. 1993. PROSEA: Vegetables. No. 8. Pudoc Scientific Publishers, Wageningen. p 23 and 43.

Tisdale, S. L. and W. L. Nelson. 1975. Soil Fertility and Fertilizers. $3^{\text {rd }}$ ed. New York: Mcmilla, 1975.

Truberg, B., and M. Huehn. 2000. Contribution to the analysis of genotype by environment interactions: Comparison of different parametric and non-parametric tests for interactions with emphasis on crossover interactions. Agron. Crop Sci.185:267-274.

Villar, M. L. D. and F. M. Valio. 1994. Rotenone and pachyrhizin in $P$. tuberosus. Effect of endogenous rotenone and pachyrhizin on early growth of Pachyrhizus tuberosus (LAM) Spreng. In Proceedings of the First International Symposium on Tuberous Legumes, ed. By Sorensen, M., The Technical Centre for Agricultural and Rural Cooperation (CTA), The Netherlands, pp. 131-143. 